The Astrophysical Journal, 611:1156-1174, 2004 August 20

(C) 2004. The American Astronomical Society. All rights reserved. Printed in U.S.A.

\title{
SPECTRAL PROPERTIES OF HEAVY IONS ASSOCIATED WITH THE PASSAGE OF INTERPLANETARY SHOCKS AT 1 AU
}

\author{
M. I. Desai and G. M. Mason ${ }^{1}$ \\ Department of Physics, University of Maryland, College Park, MD 20742 \\ M. E. WIEDENBECK \\ Jet Propulsion Laboratory, California Institute of Technology, Pasadena, CA 91109 \\ C. M. S. COHEN \\ California Institute of Technology, MC 220-47, 1200 East California Boulevard, Pasadena, CA 91125 \\ J. E. MAZUR \\ Aerospace Corporation, 2350 East El Segundo Boulevard, El Segundo, CA 90245 \\ J. R. DWYER \\ Department of Physics and Space Sciences, Florida Institute of Technology, Melbourne, FL 32901 \\ R. E. Gold and S. M. Krimigis \\ Applied Physics Laboratory, Johns Hopkins University, Laurel, MD 20723 \\ Q. $\mathrm{Hu}$ \\ Institute of Geophysics and Planetary Physics, University of California, Riverside, CA 92521 \\ C. W. SмITH \\ Institute for Earth, Oceans, and Space, University of New Hampshire, Durham, NH 03824 \\ AND \\ R. M. SKoug \\ Los Alamos National Laboratory, P.O. Box 1663, Los Alamos, NM 87545 \\ Received 2003 December 5; accepted 2004 April 26
}

\begin{abstract}
We have surveyed the energy spectra of $\sim 0.1-100 \mathrm{MeV}$ nucleon ${ }^{-1} \mathrm{C}, \mathrm{O}$, and Fe nuclei associated with the passage of 72 interplanetary (IP) shocks observed on board the $A C E$ spacecraft during the period 1997 October2002 October. Our main results are as follows: (1) The spectral fit parameters are independent of the local shock properties. (2) About 7\% of the events exhibit increasing $\mathrm{Fe} / \mathrm{O}$ ratios with energy; the remaining events have $\mathrm{Fe} / \mathrm{O}$ ratios that either remain constant or decrease with energy. (3) The Fe/O ratio in the shock-associated particles is typically $\sim 30 \%$ lower than in the ambient population. (4) The fractionation pattern of the elemental abundances, the $\mathrm{O}$ spectra, and the energy-dependence of $\mathrm{Fe} / \mathrm{O}$ at the IP shocks are remarkably similar to those of the ambient interplanetary suprathermal ion population. We suggest that the IP shocks studied here reaccelerate energetic particle seed spectra composed of ions from impulsive and gradual solar energetic particle events by systematic rigidity-dependent mechanisms in which higher rigidity ions are accelerated less efficiently than lower rigidity ions.
\end{abstract}

Subject headings: acceleration of particles — interplanetary medium — shock waves

\section{INTRODUCTION}

The acceleration of energetic particles at collisionless shocks occurs routinely in a variety of astrophysical environments both within and beyond our solar system. Common examples include heavy ions at the Earth's bow shock (e.g., Mason et al. 1996), corotating and transient interplanetary (IP) shocks (e.g., Desai et al. 1999, 2003), solar energetic particle (SEP) events produced in coronal mass ejection (CME) driven coronal shocks (e.g., Reames 1999), the solar wind termination shock (e.g., Cummings et al. 2002), and supernova remnants (e.g., Jones \& Ellison 1991).

Presently it is believed that particles gain energy at shocks most efficiently either via the first-order Fermi mechanism by

\footnotetext{
${ }^{1}$ Also at Institute for Physical Science and Technology, University of Maryland.
}

being scattered between magnetic inhomogeneities (Alfvén waves) that are convected by converging flows on either side of the shock or via the shock-drift mechanism by drifting along the shock front parallel to the $\boldsymbol{V} \times \boldsymbol{B}$ electric field (e.g., Jokipii 1982; Lee 1983; Decker 1988). Although these mechanisms have been studied extensively and incorporated within the framework of diffusive shock acceleration theory, the identity of the seed particles, the manner in which they are injected into the acceleration process, and the mechanisms that limit the acceleration processes have remained controversial (e.g., Eichler 1981; Jokipii 1987; Lee \& Fisk 1982; Forman \& Webb 1985; Jones \& Ellison 1991).

Interplanetary space near $1 \mathrm{AU}$ serves as an astrophysical laboratory where shock acceleration theories can be tested using in situ measurements of energetic ion intensity enhancements that are often observed in association with CMEdriven IP shocks (e.g., Armstrong et al. 1985; Richter et al. 
1985; Scholer 1985). The fact that CMEs propagate in the solar wind combined with a lack of detailed composition measurements from the solar wind through the energetic particle $(\sim 1 \mathrm{MeV})$ energy range led many researchers to conclude that such enhancements, also known as "energetic storm particle" or ESP events, occurred as a result of diffusive shock acceleration of solar wind ions (e.g., Lee 1983; Baring et al. 1997). Others have pointed out that the suprathermal tail of the solar wind may be the source (e.g., Gosling et al. 1981; Tsurutani \& Lin 1985).

In diffusive shock acceleration theory, the acceleration of a monoenergetic seed population results in a power-law with a spectral index $\gamma$ that is independent of ion species and determined solely by the shock compression ratio, while effects that limit the acceleration process, such as the finite width of the shock, escape of ions from shock, and/or finite acceleration time, produce a characteristic exponential rollover with $e$-folding energy $E_{0}$ (e.g., Jones \& Ellison 1991; Lee 2000; Li et al. 2003; Ruffolo \& Channok 2003). These limiting processes are assumed to depend on the diffusion coefficient, which increases with ion rigidity, such that higher rigidity ions are accelerated less efficiently than lower rigidity ions. The differential intensity $j(E)$ as a function of energy $E$ is given by $j(E)=j_{0} E^{-\gamma} \exp \left(-E / E_{0}\right)$, where $j_{0}$ is the normalization constant (e.g., Jones \& Ellison 1991). Indeed, this spectral form (hereafter referred to as the Jones \& Ellison expression) has been successful in representing the energy spectra of (1) protons, electrons, and $\alpha$-particles during several impulsive SEP events (Ellison \& Ramaty 1985), (2) ${ }^{4} \mathrm{He}-\mathrm{Fe}$ ions during a large gradual SEP event (Tylka et al. 2000), and (3) $0.1-2.0 \mathrm{MeV}$ nucleon ${ }^{-1} \mathrm{Fe}$ and $\mathrm{O}$ ions during three IP shock events (Klecker et al. 2003). Further, evidence for the occurrence of rigidity-dependent acceleration at IP shocks has been found by Klecker et al. (1981, 2000, 2003), Tylka et al. (1999), and Desai et al. (2003).

However, the above picture regarding the solar wind origin of the seed population for CME-driven IP shocks has been based on limited spectral and composition measurements. We have recently shown that the seed population for 72 such IP shocks was highly variable and composed predominantly of suprathermal ions originating from impulsive and gradual SEP events (Desai et al. 2001, 2003). In this work we survey the spectral properties of $\mathrm{C}, \mathrm{O}$, and $\mathrm{Fe}$ nuclei associated with the passage of the 72 IP shocks listed in Desai et al. (2003) over a significantly broader energy range $\left(0.1-100 \mathrm{MeV}\right.$ nucleon $\left.^{-1}\right)$ than previously available. In particular, we investigate whether the spectral indices of the different species are similar and examine the relationship between the spectral properties and the strength of the IP shocks. We also investigate the role of a variable suprathermal seed population and the possible occurrence of rigidity-dependent fractionation processes in individual events.

\section{INSTRUMENTATION}

This study uses instrumentation on board the Advanced Composition Explorer ( $A C E$ ) spacecraft, which was launched in 1997 August (Stone et al. 1998a) to orbit around the sunward Lagrangian point. Energetic particle measurements from $\sim 0.1$ to a few $\mathrm{MeV}$ nucleon ${ }^{-1}$ were obtained by the Ultra Low Energy Isotope Spectrometer (ULEIS), which is a time-offlight mass spectrometer with a geometry factor of $\sim 1 \mathrm{~cm}^{2} \mathrm{sr}$ and a $50 \mathrm{~cm}$ flight path (Mason et al. 1998). Measurements between $\sim 5$ and $100 \mathrm{MeV}$ nucleon $^{-1}$ were obtained by the
Solar Isotope Spectrometer (SIS), which is a multidetector $d E / d x$ versus residual energy spectrometer with two telescopes and a geometry factor of $\sim 38 \mathrm{~cm}^{2} \mathrm{sr}$ (Stone et al. 1998b). Both sensors were designed to achieve sensitivity and mass resolution that exceeded those of previous instruments in similar energy ranges.

In order to compute the combined energy spectra measured by ULEIS and SIS, we checked the intercalibration of the two instruments by comparing the fluences measured during the shock-associated sampling intervals for the 20 events listed in Table 1. With the exception of portions of three events where ULEIS partially saturated, we found that the $\mathrm{C}$ and $\mathrm{O}$ fluences were in excellent agreement at the point where the energy coverage of the two instruments overlaps, thereby verifying the intercalibration of the two instruments.

We also used solar wind plasma and magnetic field measurements obtained respectively by the Solar Wind Electron Proton and Alpha Monitor (SWEPAM; McComas et al. 1998) and the magnetometer (MAG; Smith et al. 1998) on board $A C E$ to identify the arrival of the IP shocks and determine various parameters that characterize their relative strengths.

\section{SELECTION OF INTERPLANETARY SHOCK EVENTS AND THEIR SAMPLING INTERVALS}

\subsection{Example of an IP Shock Event Measured by ULEIS}

The 72 IP shock events surveyed here were observed at $A C E$ from 1997 October through 2002 September (see Table A1 in the Appendix for the shock arrival times at $A C E$ ). These IP shock events were also studied by Desai et al. (2003), who had identified $\sim 1-5$ day intervals (listed in their Table 1) for measuring energetic ions associated with the passage of each shock at $1 \mathrm{AU}$ on the basis of the following criteria: (1) The 0.5-2.0 MeV nucleon ${ }^{-1}{ }^{4} \mathrm{He}, \mathrm{O}$, and Fe intensities should increase by at least a factor of 5 within a $24 \mathrm{hr}$ period centered on the arrival of the IP shock. (2) The $0.5-2.0 \mathrm{MeV}_{\text {nucleon }}{ }^{-1}$ intensity-time profiles of ${ }^{4} \mathrm{He}, \mathrm{O}$, and $\mathrm{Fe}$ should be generally similar. (3) The $0.3-3.0 \mathrm{MeV}$ nucleon ${ }^{-1} \mathrm{Fe}$-group ions should not exhibit velocity dispersion. In the current study, the emphasis is on determining the heavy-ion spectra over the broadest feasible energy range. Because of the rarity of the heavy ions and steepness of the spectra, this requires a long averaging time. We have adopted the approach here of averaging the spectra over the entire event, as is commonly done in spectral and composition studies of solar energetic particles. We recognize that such event averages will necessarily add together particles with different acceleration and transport histories, and it will be necessary to keep this in mind when interpreting the data.

As an example of ULEIS observations, Figure $1 a$ displays the hourly averaged time-intensity profiles of $\mathrm{C}, \mathrm{O}$, and $\mathrm{Fe}$ nuclei for event 13 in Desai et al. (2003) and Table A1. The ULEIS data in Figure $1 a$ are presented in two energy intervals, namely, $0.16-0.23$ and $0.91-1.28 \mathrm{MeV}$ nucleon $^{-1}$. Figures $1 b$ and $1 c$ respectively show the temporal evolution of $\mathrm{C} / \mathrm{O}$ and $\mathrm{Fe} / \mathrm{O}$ ratios at the above two energies $(\sim 0.2$ and $\sim 1 \mathrm{MeV}$ nucleon $^{-1}$ ), while Figures $1 d$ and $1 e$ respectively show 5 minute averages of the magnetic field magnitude and solar wind speed from 1999 June 22 through June 29. Two IP shocks were observed at $A C E$ on 1999 June 26, and their arrivals are identified by the abrupt increases in $|B|$ and $V$ at 0218 UT (S1) and 1920 UT (S2; event 13).

Figure 1 shows that the $\sim 0.2 \mathrm{MeV}$ nucleon $^{-1} \mathrm{C}, \mathrm{O}$, and $\mathrm{Fe}$ intensities exhibited a small abrupt increase in association 
TABLE 1

Interplanetary Shock Events Measured by ULEIS and SIS

\begin{tabular}{|c|c|c|c|c|c|c|}
\hline Number & $\begin{array}{c}\text { Event } \\
\text { Number }^{\text {a }}\end{array}$ & $\begin{array}{c}\text { Shock Arrival } \\
\text { Time at } A C E^{\mathrm{a}} \\
\text { (UT) }\end{array}$ & $\begin{array}{l}\text { ULEIS Sampling } \\
\text { Times }^{\mathrm{a}} \\
\text { (UT) }\end{array}$ & $\begin{array}{l}\text { SIS Sampling } \\
\text { Times }^{\mathrm{b}} \\
\text { (UT) }\end{array}$ & $\begin{array}{l}\text { ULEIS Fe/O } \\
0.11-0.32 \mathrm{MeV} \\
\text { nucleon }^{-1}\end{array}$ & $\begin{array}{c}\text { SIS Fe/O } \\
12-60 \mathrm{MeV} \\
\text { nucleon }^{-1}\end{array}$ \\
\hline \multicolumn{7}{|c|}{1998} \\
\hline 1 . & $6^{\mathrm{c}}$ & Sep 24, 2313 & $\begin{array}{l}\text { Sep 24, 1717-Sep 24, } 2315 \\
\text { Sep 25, 0158-Sep 25, } 0818\end{array}$ & Sep 24, 1442-Sep 25, 0754 & $0.147 \pm 0.003$ & $0.141 \pm 0.051$ \\
\hline \multicolumn{7}{|c|}{1999} \\
\hline 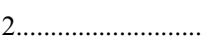 & 12 & May 5, 1459 & May 5, 1213-May 6, 2346 & May 5, 0549-May 5, 2334 & $0.116 \pm 0.007$ & $<0.032^{\mathrm{d}}$ \\
\hline 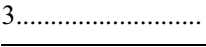 & 13 & Jun 26, 1925 & Jun 25, 0844-Jun 27, 1257 & Jun 26, 0029-Jun 27, 0111 & $0.164 \pm 0.017$ & $\cdots$ \\
\hline \multicolumn{7}{|c|}{2000} \\
\hline 4 & $24^{\mathrm{c}}$ & Feb 11, 2319 & $\begin{array}{l}\text { Feb 11, 1244-Feb 11, } 2319 \\
\text { Feb 12, 0226-Feb 12, } 1906\end{array}$ & Feb 11, 1343-Feb 12, 0210 & $0.146 \pm 0.014$ & $\ldots$ \\
\hline 5 & 30 & Jul 13, 0919 & Jul 12, 1951-Jul 14, 1002 & Jul 13, 0315-Jul 13, 1653 & $0.1 \pm 0.004$ & $0.112 \pm 0.031$ \\
\hline 6............................ & 31 & Jul 19, 1449 & Jul 19, 0453-Jul 20, 0306 & Jul 19, 0922-Jul 20, 0245 & $0.11 \pm 0.002$ & $0.054 \pm 0.007$ \\
\hline 7.......................... & 35 & Aug 11, 1811 & Aug 10, 1737-Aug 12, 1439 & Aug 11, 0800-Aug 11, 2300 & $0.175 \pm 0.017$ & $\ldots$ \\
\hline $8 \ldots \ldots \ldots \ldots \ldots \ldots \ldots \ldots \ldots$ & 39 & Nov 4, 0135 & Nov 3, 0755-Nov 5, 1102 & Nov 3, 1302-Nov 4, 2225 & $0.164 \pm 0.019$ & $\cdots$ \\
\hline \multicolumn{7}{|c|}{2001} \\
\hline 9 . & 43 & Mar 27, 1716 & Mar 25, 1806-Mar 28, 0120 & Mar 27, 1256-Mar 27, 2300 & $0.128 \pm 0.003$ & $\cdots$ \\
\hline 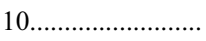 & 47 & Apr 7, 1659 & Apr 7, 0706-Apr 8, 0425 & Apr 7, 1022-Apr 7, 2324 & $0.108 \pm 0.008$ & $\ldots$ \\
\hline 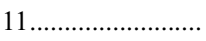 & 48 & Apr 8, 1033 & Apr 8, 0426-Apr 8, 1719 & Apr 8, 0537-Apr 8, 1728 & $0.176 \pm 0.008$ & $\ldots$ \\
\hline $12 \ldots \ldots \ldots \ldots \ldots \ldots \ldots$ & $50^{\mathrm{c}}$ & Apr 28, 0432 & $\begin{array}{l}\text { Apr 27, 1519-Apr 28, } 0337 \\
\text { Apr 28, 1337-Apr 29, } 0342\end{array}$ & Apr 27, 1758-Apr 28, 0800 & $0.222 \pm 0.031$ & $\ldots$ \\
\hline $13 \ldots \ldots \ldots \ldots \ldots \ldots \ldots$ & 54 & Sep 14, 0118 & Sep 13, 2226-Sep 16, 2053 & Sep 13, 2020-Sep 14, 0958 & $0.118 \pm 0.008$ & $<0.130^{\mathrm{d}}$ \\
\hline 14 & 59 & Nov 6,0124 & Nov 5, 2253-Nov 6, 2008 & Nov $5,1325-$ Nov 6,1915 & $0.201 \pm 0.019$ & $0.069 \pm 0.002$ \\
\hline $15 \ldots \ldots \ldots \ldots \ldots \ldots \ldots \ldots$ & 60 & Nov 19,1735 & Nov 19, 1213-Nov 20, 1706 & Nov 19, 1057-Nov 20, 0742 & $0.209 \pm 0.003$ & $0.075 \pm 0.033$ \\
\hline $16 \ldots \ldots \ldots \ldots \ldots \ldots \ldots \ldots$ & 61 & Nov 24, 0538 & Nov 23, 2232-Nov 24, 2113 & Nov 23, 1909-Nov 24, 1951 & $0.397 \pm 0.002$ & $0.087 \pm 0.002$ \\
\hline \multicolumn{7}{|c|}{2002} \\
\hline 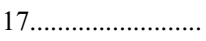 & 66 & Apr 19, 0803 & Apr 18, 1840-Apr 19, 1359 & Apr 19, 0303-Apr 19, 1343 & $0.402 \pm 0.049$ & $0.046 \pm 0.020$ \\
\hline $18 \ldots \ldots \ldots \ldots \ldots \ldots \ldots \ldots$ & 69 & May 23, 1016 & May 23, 0524-May 23, 1417 & May 23, 0613-May 23, 1343 & $0.230 \pm 0.011$ & $0.025 \pm 0.002$ \\
\hline $19 \ldots \ldots \ldots \ldots \ldots \ldots \ldots$ & 70 & Jul 17, 1526 & Jul 17, 1306-Jul 17, 2106 & Jul 17, 1256-Jul 17, 2050 & $0.213 \pm 0.009$ & $0.046 \pm 0.007$ \\
\hline $20 \ldots \ldots \ldots \ldots \ldots \ldots \ldots$ & 71 & Sep 7, 1609 & Sep 7, 1319-Sep 8, 0302 & Sep 7, 1208-Sep 7, 2237 & $0.355 \pm 0.012$ & $0.034 \pm 0.004$ \\
\hline
\end{tabular}

a Taken from Table 1 of Desai et al. (2003).

b See text for details of selection of SIS sampling intervals.

${ }^{c}$ Events with partial saturation effects in ULEIS.

d $1 \sigma$ upper limits are provided for events with relative uncertainty greater than $50 \%$.

with the arrival of the first IP shock, while the second shock was associated with intensity enhancements between $\sim 2$ and 3 orders of magnitude at both energies. Thus, the ion population measured during the entire shock-associated sampling interval identified by Desai et al. (2003) is essentially dominated by ions associated with the second IP shock. The figure also shows that the $\mathrm{C} / \mathrm{O}$ ratios at both energies remained at $\sim 0.4$ during the 7 day interval. In contrast, the $\sim 0.2 \mathrm{MeV}$ nucleon ${ }^{-1} \mathrm{Fe} / \mathrm{O}$ ratio was highly variable throughout this period; the average $\mathrm{Fe} / \mathrm{O}$ at $\sim 0.2 \mathrm{MeV}$ nucleon $^{-1}$ was $\sim 0.5$ during the ambient interval from June 221035 UT through June 241805 UT, dropped to $\sim 0.2$ during the shock-associated interval from June 250844 UT through June 271257 UT, and increased to $\sim 1$ from June 28 through June 29. The average $\mathrm{Fe} / \mathrm{O}$ at $\sim 1 \mathrm{MeV}$ nucleon $^{-1}$ during the shock sampling interval was less than 0.1 . In summary, the $\mathrm{C} / \mathrm{O}$ ratios during the shock-associated interval were similar to those measured in the surrounding interplanetary medium, while the $\mathrm{Fe} / \mathrm{O}$ ratios at both energies decreased around shock passage, the decrease being significantly larger at higher energy.
We first remark that IP shocks, such as S1 in Figure 1, with relatively minor effects on the energetic particles were also observed during 14 other sampling intervals of Desai et al. (2003). In this survey we consider that the ion populations measured during these intervals were associated with the major IP shocks whose arrival times are given in Table A1. Figure 1 shows that the heavy-ion intensity-time profiles exhibited a remarkable degree of complexity, particularly during the shock-associated sampling interval. Although this was also true for most of the events in our survey, we remark that the intensity enhancements observed by ULEIS during these long intervals were essentially dominated by ion populations associated with the arrival of the IP shocks at $A C E$. Complex intensity-time profiles such as these are also commonly observed in studies of protons at IP shocks (van Nes et al. 1984; Kallenrode 1995).

To compare properties of the shock-associated ion population with those of the highly variable suprathermal ion population ubiquitous in the interplanetary medium (see Tsurutani \& Lin 1985; Mason 2000; Wiedenbeck et al. 2003), Desai 

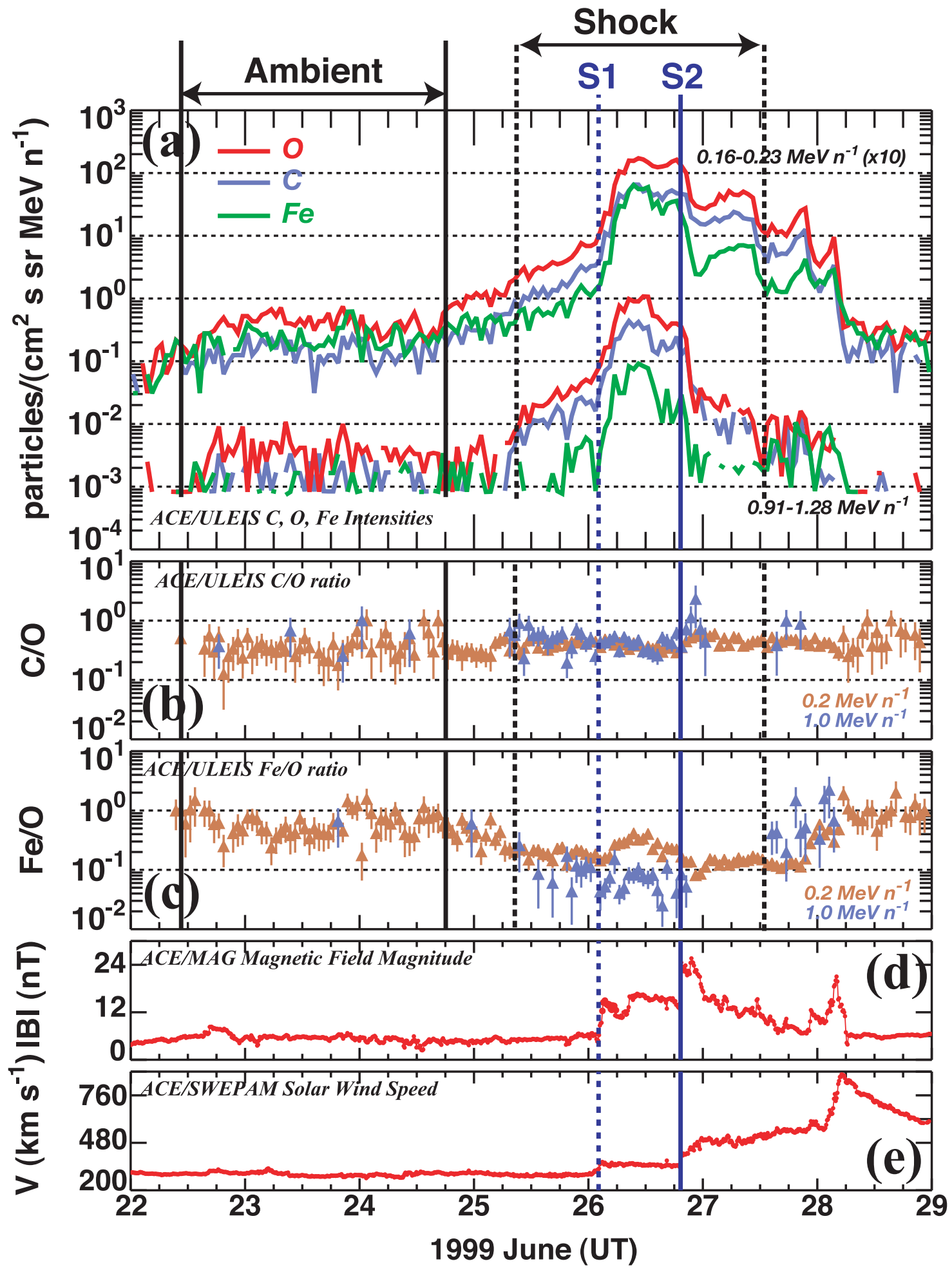

FIG. 1.- Hourly averages of $(a) 0.16-0.23$ and $0.91-1.28 \mathrm{MeV}$ nucleon $^{-1} \mathrm{C}, \mathrm{O}$, and Fe intensities, $(b) \mathrm{C} / \mathrm{O}$ ratios, and $(c) \mathrm{Fe} / \mathrm{O}$ ratios. Also shown are 5 minute averages of $(d)$ the magnetic field magnitude $B$ and $(e)$ the solar wind speed $V$ from 1999 June 22 through 29 . The blue vertical lines S1 and S2 (event 13 ; see text for details) mark the arrival of IP shocks at $A C E$ at 0218 and 1920 UT on 1999 June 26, respectively. Dashed black vertical lines: Time interval for measuring shockassociated energetic ions. Solid black vertical lines: Time interval for measuring ambient energetic ions in the interplanetary medium.

et al. (2003) also identified 72 intervals prior to the start of the shock-associated ramp-up of the intensities corresponding to each IP shock in the survey (e.g., the ambient interval in Fig.1). Desai et al. (2003) referred to these intervals as "upstream" sampling times and to the associated greater than $0.1 \mathrm{MeV}$ nucleon $^{-1}$ ion population as the "upstream" ion population. Strictly speaking, however, this terminology is misleading because intervals containing the shockassociated ramp-up of the intensities have also often been referred to as upstream intervals (e.g., Lee 1983; Kennel et al. 1986). Owing to this as well as to avoid possible confusion with the "upstream" intervals used to estimate the shock 


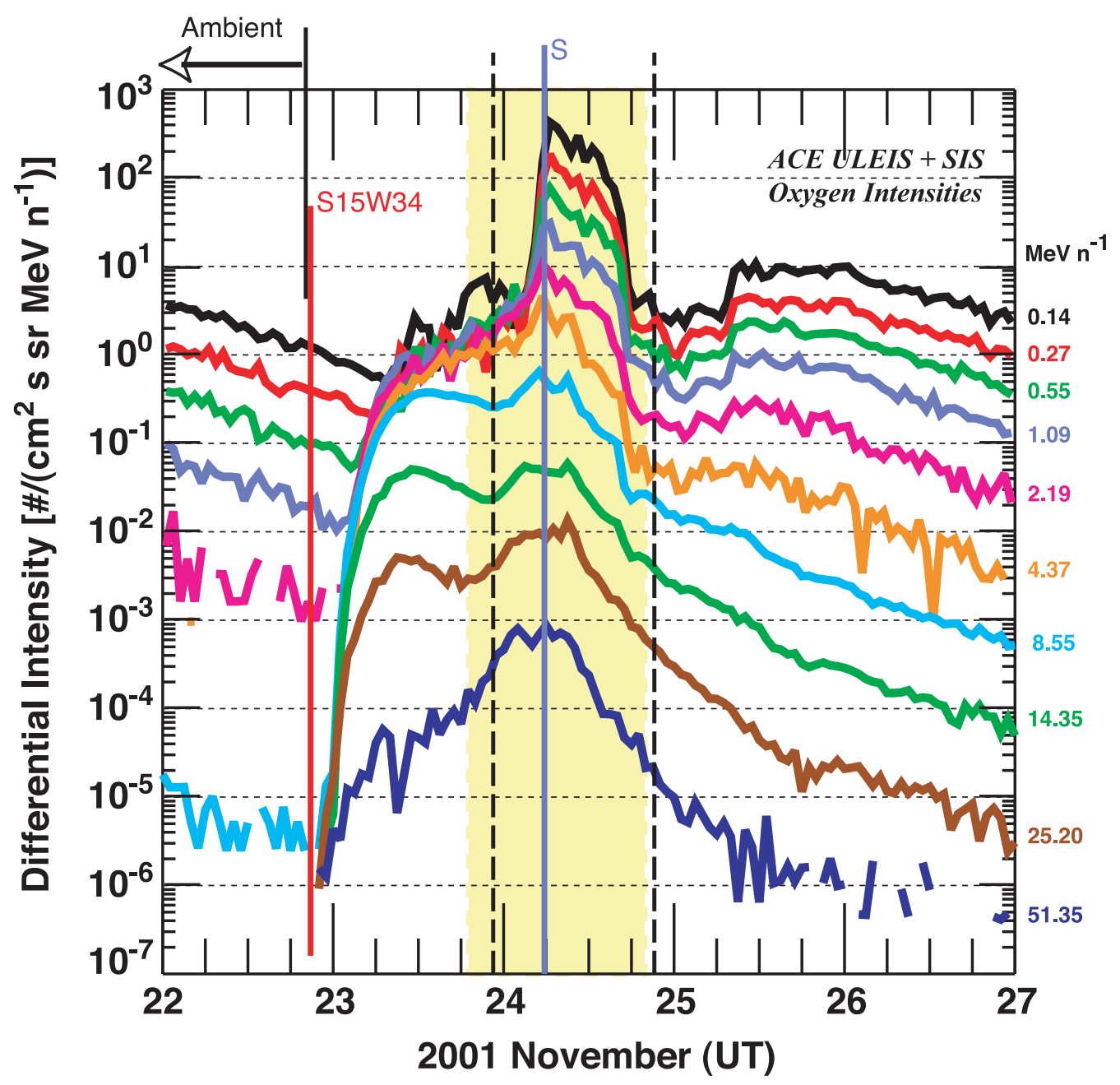

FIG. 2.- Hourly averaged O intensities between $\sim 0.15$ and $50 \mathrm{MeV}$ nucleon ${ }^{-1}$ measured by ULEIS and SIS for the period 2001 November $22-27$. The arrival of IP shock event 61 is marked S. Red line: Time of flare in AR 9704 at $515^{\circ}, \mathrm{W} 34^{\circ}$. Dashed black lines: Sampling interval for ULEIS. Shaded region: Sampling interval for SIS.

strength parameters in $\S 4.2$, hereafter we refer to the upstream sampling times of Desai et al. (2003) as the "ambient" sampling intervals and to the corresponding greater than $0.1 \mathrm{MeV}$ nucleon $^{-1}$ energetic ions as the "ambient" suprathermal ion population.

As discussed in Desai et al. (2003), however, the main limitation of using the greater than $0.1 \mathrm{MeV}^{\text {nucleon }}{ }^{-1}$ ions measured at $A C E$ during the ambient intervals as possible proxies for the source population for IP shocks is that these ions do not in fact interact with the shocks in our study. However, each of these preceding intervals was selected to fall within $\mathrm{a} \sim 7$ day period prior to the start of the shock-associated interval to provide a reasonable chance of measuring the properties of the ambient suprathermal population that the IP shocks may have encountered en route to $1 \mathrm{AU}$. This approach assumes that the suprathermal population is correlated over a period of days, and although this is plausible given the timescale of many interplanetary events, it is clearly an approximation at best. This hypothesis was justified to a certain extent by the results of Desai et al. (2003), who showed that the $0.5-$ 2.0 $\mathrm{MeV}$ nucleon $^{-1} \mathrm{Fe} / \mathrm{O}$ ratios as well as the average elemental abundances from ${ }^{4} \mathrm{He}$ through $\mathrm{Fe}$ at the IP shocks were reasonably well correlated with those measured during the ambient intervals (see also Tan et al. 1989). In this paper we explore the relationship between the spectral properties of the shock-associated heavy ions and those measured during the ambient sampling intervals.

\subsection{Example of an IP Shock Event Measured by ULEIS and SIS}

For all 72 shocks, we also surveyed the greater than $7 \mathrm{MeV}$ nucleon ${ }^{-1}$ hourly averaged time-intensity profiles of $\mathrm{O}$ nuclei measured by SIS. We identified 20 events for which the higher energy intensity enhancements were dominated by particle populations associated with the passage of the IP shocks. Figure 2 displays the time-intensity profiles of $\sim 0.15-$ $50 \mathrm{MeV}$ nucleon $^{-1} \mathrm{O}$ ions measured by ULEIS and SIS in association with the passage of IP shock event 61 (S), which arrived at $A C E$ at $0538 \mathrm{UT}$ on 2001 November 24. The associated solar activity on November 22 may be summarized as follows (see, e.g., Dalla et al. 2003): The GOES spacecraft recorded an M3.8 X-ray flare at 2018 UT from NOAA Active Region 9678 at $\mathrm{S} 25^{\circ}$, W67 ${ }^{\circ}$. Later, two flares (C5.9 and M9.9) were observed at 2158 and 2232 UT, respectively, from AR 9704 at $\mathrm{S} 15^{\circ}, \mathrm{W} 34^{\circ}$. The $\mathrm{SOHO}$ spacecraft observed two halo CMEs at 2058 and 2330 UT with speeds of $\sim 1246$ and $\sim 1500 \mathrm{~km} \mathrm{~s}^{-1}$, respectively, in association with the two M-class flares. 
Figure 2 shows that the less than $5 \mathrm{MeV}$ nucleon $^{-1} \mathrm{O}$ intensities between 0000 and 2230 UT on November 22 were dominated by the decay of a previous IP shock event (event 60 in our survey). From $\sim 2230$ UT on November 22 , the O intensities above $\sim 1 \mathrm{MeV}$ nucleon ${ }^{-1}$ increased dramatically and exhibited velocity dispersion during the onset of an SEP event that was probably associated with the flares and halo $\mathrm{CME}$ from $\mathrm{AR} 9704$ at $\mathrm{S} 15^{\circ}, \mathrm{W} 34^{\circ}$. Although the intensities at all energies increased by 1-2 orders of magnitude and peaked near the IP shock (S), with the shock-associated population being superposed on the SEP event, we note that the time histories above and below $\sim 5 \mathrm{MeV}$ nucleon $^{-1}$ exhibited different behavior around shock passage. In particular, the greater than $5 \mathrm{MeV}$ nucleon $^{-1}$ intensities had broad $\sim 18-$ $24 \mathrm{hr}$ peaks centered on the shock, whereas the less than $5 \mathrm{MeV}$ nucleon $^{-1}$ intensities increased abruptly around $3 \mathrm{hr}$ prior to the arrival of the shock and peaked at the shock. Then the lower energy intensities decreased slowly by about a factor of 5 over a $12 \mathrm{hr}$ period and dropped abruptly by about an order of magnitude probably because of the arrival of the interplanetary counterpart of the CME at $A C E$ (Cane \& Richardson 2003).

In general, the shock-associated sampling intervals identified by Desai et al. (2003) using the lower energy $(<2.0 \mathrm{MeV}$ nucleon $^{-1}$ ) ULEIS measurements either did not contain the entire IP shock-associated population at SIS energies or contained particle populations associated with preceding or simultaneous SEP events (also see Mason et al. 1999b; Slocum et al. 2003). Consequently, we selected new sampling intervals to compute the energy spectra above $\sim 7 \mathrm{MeV}$ nucleon $^{-1}$ for these 20 events. These intervals, along with the $\mathrm{Fe} / \mathrm{O}$ ratios at $0.11-0.32$ and $12-60 \mathrm{MeV}$ nucleon $^{-1}$, are listed in Table 1. Ten of the shocks had finite $12-60 \mathrm{MeV}^{\text {nucleon }}{ }^{-1}$ ratios, while the rest had insufficient statistics or did not show Fe intensity enhancements associated with the shock passage.

\section{DATA ANALYSIS}

\subsection{Spectral Fitting}

In order to survey the spectral properties of heavy ions associated with the passage of the 72 IP shocks at $A C E$, we fitted the $\sim 0.1-5.0 \mathrm{MeV}$ nucleon ${ }^{-1}$ differential energy spectra of $\mathrm{C}, \mathrm{O}$, and Fe nuclei measured by ULEIS with the Jones \& Ellison expression. Throughout this survey we have only included ULEIS data points with a relative uncertainty of less than $35 \%$. The spectrum for each species was fitted independently using a nonlinear least-squares technique that minimized the $\chi^{2}$ and yielded values for $j_{0}, \gamma$, and $E_{0}$. However, a serious limitation of using the $\gamma$-values from these fits to characterize the lower energy $\left(\leq 0.5 \mathrm{MeV}\right.$ nucleon $\left.^{-1}\right)$ part of the spectrum arises because $\gamma$ and $E_{0}$ are not orthogonal but coupled (also see Mewaldt et al. 2003). Thus, the fitted value for the low-energy power-law index $\gamma$ in fact depends on the relative steepness of the higher energy portion (typically above $0.5 \mathrm{MeV}$ nucleon $^{-1}$ ) of the spectrum. However, because of the $M / Q$-dependent fractionation effects of processes that limit the acceleration mechanisms, the values of $E_{0}$ for many events were significantly lower for $\mathrm{Fe}$ than those for $\mathrm{C}$ and $\mathrm{O}$ (e.g., see event 13 in $\S 5.1$ ). This yielded significantly different values of $\gamma$ for $\mathrm{Fe}$ when compared with those of $\mathrm{C}$ and $\mathrm{O}$, even though the lower energy portions of the spectra appeared to have similar slopes. Thus, in order to survey the properties of the lower energy portions of the spectra independently of their behavior at higher energies, we also fitted the $0.1-0.5 \mathrm{MeV}$ nucleon $^{-1}$ ULEIS intensities with a power-law of the form $j(E)=j_{0} E^{-\gamma}$.

Table 2 lists the values of $\gamma$ obtained from power-law fits to the lower energy portions of the spectra, while those of $E_{0}$ and the reduced $\chi^{2}, \chi_{v}^{2}$ ( $v$ is the number of degrees of freedom), are obtained from fitting the energy spectra between $\sim 0.1$ and 5.0 MeV nucleon ${ }^{-1}$ with the Jones \& Ellison expression. In general, the fits to the ULEIS energy spectra for all events were excellent visually and typically had $\sim 50 \%$ probabilities for the goodness-of-fit statistic (from Bevington \& Robinson 1992; also see Klecker et al. 2003). This also indicates that the IP shock-associated heavy-ion spectra do not exhibit spectral breaks (i.e., are not represented by two power laws), but are relatively smooth over the ULEIS energy range. Finally, we also fitted the ULEIS and SIS energy spectra with the Jones \& Ellison expression for the 20 events listed in Table 1. In general, we found that the fits for many of these events were visually poor and also gave very high $\chi_{v}^{2}$ values.

\subsection{Estimating the Shock Strength Parameters}

For each of the 72 IP shocks in the survey we used the highresolution (64 s) SWEPAM and MAG data sets to identify three consecutive data points upstream and downstream of the shocks during which the solar wind speed and magnetic field magnitude remained relatively stable. We then employed the nonlinear least-squares technique of Szabo (1994) to simultaneously solve the complete set of Rankine-Hugoniot (R-H) relations for the nine pairs of upstream and downstream data points and obtain unique values for the various shock strength parameters. The error estimate for each parameter was obtained by propagating constant values for the errors associated with each individual data point; uncertainties were taken as $0.1 \mathrm{nT}$ for magnetic vectors, $1 \mathrm{~cm}^{-3}$ for density, and $40 \mathrm{~km} \mathrm{~s}^{-1}$ for the velocity. This particular technique is a significant improvement upon both preaveraged coplanarity methods (e.g., Tsurutani \& Lin 1985) as well as the Viñas \& Scudder (1986) method, which also solved the R-H relations but without including the plasma temperature measurements (see Szabo 1994 for a detailed comparison of the results from the three methods).

One limitation of the present analysis, however, is that instead of using the actual electron temperature measurements we assigned a constant typical value to each individual data point upstream and downstream of a shock. Nonetheless, preliminary case studies indicate that using the real electron temperature measurements do not change the final results significantly. In addition, we also used the magnetic coplanarity technique (Tsurutani \& Lin 1985) to determine the $\theta_{B n}$ values for 11 events where the SWEPAM proton data were unavailable. Table A1 lists the Mach number $M_{A}$, the magnetic $M$ and density $H$ compression ratios, the shock normal angle $\theta_{B n}$, and the shock speeds $V_{\mathrm{IP}}$ and $V_{S}$ in the spacecraft and upstream plasma frames, respectively, for the 72 events studied here.

\section{RESULTS OF SPECTRAL FITS}

\subsection{Variability in Shock-averaged Spectra Measured by ULEIS}

The left panels of Figure 3 display the C, O, and Fe energy spectra measured by ULEIS for three representative IP shocks, namely, events 13,18 , and 37 . The $\mathrm{C}, \mathrm{O}$, and Fe spectra for all three events were well fitted by the Jones \& Ellison expression. The value of $E_{0}$ for $\mathrm{Fe}$ in event 13 was lower by about a factor of 2 than those for $\mathrm{C}$ and $\mathrm{O}$ (see Table 2), leading to a 
TABLE 2

Spectral Fit Parameters for C, O, and Fe during the 72 Interplanetary Shocks in This Survey

\begin{tabular}{|c|c|c|c|c|c|c|c|c|c|}
\hline \multirow[b]{2}{*}{ NuMBer } & \multicolumn{3}{|c|}{ CARBON } & \multicolumn{3}{|c|}{ OXYGEN } & \multicolumn{3}{|c|}{ IRON } \\
\hline & $\gamma^{\mathrm{a}}$ & $E_{0}^{\mathrm{b}, \mathrm{c}}$ & $\chi_{v}^{2}$ & $\gamma^{\mathrm{a}}$ & $E_{0}^{\mathrm{b}, \mathrm{c}}$ & $\chi_{v}^{2}$ & $\gamma^{\mathrm{a}}$ & $E_{0}^{\mathrm{b}, \mathrm{c}}$ & $\chi_{v}^{2}$ \\
\hline$\ldots \ldots \ldots . .$. & $2.45 \pm 0.03$ & $0.19 \pm 0.11$ & 0.46 & $2.61 \pm 0.15$ & $0.4 \pm 0.08$ & 0.87 & $2.44 \pm 0.32$ & & 0.69 \\
\hline 2 & $2.71 \pm 0.34$ & $0.64 \pm 0.23$ & 0.82 & $2.58 \pm 0.09$ & $0.83 \pm 0.23$ & 0.88 & $2.36 \pm 0.07$ & & 0.88 \\
\hline 3...................... & $2.2 \pm 0.28$ & $0.65 \pm 0.09$ & 0.88 & $2.03 \pm 0.08$ & $0.62 \pm 0.06$ & 0.88 & $2.16 \pm 0.15$ & $1.0 \pm 0.39$ & 0.89 \\
\hline ............. & $1.56 \pm 0.35$ & $0.79 \pm 0.12$ & 0.9 & $1.53 \pm 0.24$ & $1.4 \pm 0.34$ & 0.91 & $2.08 \pm 0.21$ & $0.58 \pm 0.07$ & 0.9 \\
\hline $5 \ldots \ldots \ldots \ldots \ldots \ldots$ & $3.1 \pm 0.42$ & $0.57 \pm 0.27$ & 0.78 & $3.08 \pm 0.21$ & $0.47 \pm 0.11$ & 0.87 & $3.63 \pm 0.38$ & $0.12 \pm 0.04$ & 0.46 \\
\hline 6 & $1.96 \pm 0.25$ & $1.36 \pm 0.42$ & 0.89 & $1.83 \pm 0.04$ & $1.22 \pm 0.14$ & 0.9 & $1.69 \pm 0.04$ & $0.88 \pm 0.09$ & 0.89 \\
\hline 7 & $2.5 \pm 0.31$ & $0.21 \pm 0.02$ & 0.83 & $2.65 \pm 0.15$ & $0.32 \pm 0.07$ & 0.83 & $3.23 \pm 0.28$ & $0.26 \pm 0.08$ & 0.78 \\
\hline 8 & $2.12 \pm 0.16$ & $1.75 \pm 0.38$ & 0.89 & $2.09 \pm 0.07$ & $1.66 \pm 0.17$ & 0.91 & $1.74 \pm 0.12$ & $1.02 \pm 0.26$ & 0.89 \\
\hline 9.………....... & $1.92 \pm 0.03$ & $0.63 \pm 0.41$ & 0.79 & $2.06 \pm 0.04$ & $0.66 \pm 0.07$ & 0.96 & $1.99 \pm 0.13$ & $0.25 \pm 0.05$ & 0.82 \\
\hline $10 \ldots \ldots$ & $2.13 \pm 0.12$ & $0.81 \pm 0.12$ & 0.87 & $2.18 \pm 0.08$ & $0.85 \pm 0.09$ & 0.91 & $2.38 \pm 0.09$ & $0.6 \pm 0.21$ & 0.87 \\
\hline $11 \ldots \ldots \ldots \ldots \ldots$ & $2.18 \pm 0.25$ & $0.69 \pm 0.1$ & 0.88 & $2.25 \pm 0.2$ & $0.81 \pm 0.15$ & 0.88 & $2.73 \pm 0.04$ & $0.87 \pm 0.64$ & 0.83 \\
\hline$\ldots \ldots \ldots \ldots$ & $1.4 \pm 0.11$ & $1.28 \pm 0.1$ & 0.92 & $1.54 \pm 0.07$ & $1.27 \pm 0.05$ & 0.92 & $1.65 \pm 0.03$ & $0.49 \pm 0.07$ & 0.88 \\
\hline $13 \ldots \ldots \ldots \ldots \ldots$ & $1.35 \pm 0.14$ & $0.79 \pm 0.06$ & 0.9 & $1.4 \pm 0.07$ & $1.0 \pm 0.12$ & 0.91 & $2.04 \pm 0.12$ & $0.48 \pm 0.03$ & 0.91 \\
\hline 14 & $2.91 \pm 0.08$ & $0.94 \pm 0.53$ & 0.82 & $2.94 \pm 0.11$ & $\ldots$ & 0.85 & $2.7 \pm 0.06$ & $0.99 \pm 0.3$ & 0.89 \\
\hline $15 \ldots \ldots \ldots \ldots \ldots$ & $3.21 \pm 0.51$ & $\ldots$ & 0.79 & $2.82 \pm 0.07$ & $0.42 \pm 0.07$ & 0.85 & $2.87 \pm 0.09$ & $0.37 \pm 0.03$ & 0.91 \\
\hline …….... & $2.73 \pm 0.29$ & $0.38 \pm 0.06$ & 0.85 & $2.66 \pm 0.13$ & $0.48 \pm 0.06$ & 0.87 & $3.12 \pm 0.15$ & $0.82 \pm 0.75$ & 0.79 \\
\hline .......... & $2.88 \pm 0.3$ & $0.41 \pm 0.1$ & 0.8 & $2.7 \pm 0.09$ & $0.47 \pm 0.1$ & 0.87 & $2.68 \pm 0.19$ & $0.44 \pm 0.14$ & 0.84 \\
\hline 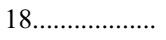 & $2.92 \pm 0.32$ & $0.51 \pm 0.22$ & 0.79 & $2.78 \pm 0.11$ & $0.51 \pm 0.17$ & 0.85 & $2.66 \pm 0.1$ & $0.44 \pm 0.15$ & 0.78 \\
\hline 19..................... & $3.21 \pm 0.23$ & $0.28 \pm 0.1$ & 0.5 & $3.1 \pm 0.02$ & $0.76 \pm 0.36$ & 0.78 & $2.73 \pm 0.08$ & $\ldots$ & 0.78 \\
\hline $20 \ldots \ldots \ldots \ldots \ldots$ & $1.74 \pm 0.16$ & $0.8 \pm 0.06$ & 0.86 & $1.79 \pm 0.05$ & $1.12 \pm 0.16$ & 0.92 & $2.08 \pm 0.06$ & $0.45 \pm 0.03$ & 0.91 \\
\hline $21 \ldots$. & $2.22 \pm 0.21$ & $0.45 \pm 0.06$ & 0.85 & $2.25 \pm 0.1$ & $0.52 \pm 0.07$ & 0.88 & $2.61 \pm 0.24$ & $0.28 \pm 0.05$ & 0.83 \\
\hline $22 \ldots$ & $2.45 \pm 0.14$ & $0.61 \pm 0.25$ & 0.84 & $2.41 \pm 0.07$ & $1.09 \pm 0.19$ & 0.9 & $2.61 \pm 0.05$ & $0.98 \pm 0.14$ & 0.86 \\
\hline $23 \ldots$ & $2.4 \pm 0.41$ & $0.42 \pm 0.09$ & 0.83 & $2.45 \pm 0.27$ & $0.59 \pm 0.13$ & 0.89 & $3.22 \pm 0$ & $0.45 \pm 0.11$ & 0.83 \\
\hline $24 \ldots$ & $2.48 \pm 0.19$ & $1.22 \pm 0.4$ & 0.86 & $2.34 \pm 0.15$ & $1.09 \pm 0.22$ & 0.92 & $2.57 \pm 0.07$ & $0.96 \pm 0.28$ & 0.88 \\
\hline $25 \ldots \ldots \ldots \ldots \ldots$ & $3.69 \pm 0.51$ & $\ldots$ & 0.84 & $3.46 \pm 0.09$ & $\ldots$ & 0.9 & $2.99 \pm 0.03$ & $\ldots$ & 0.91 \\
\hline $26 \ldots \ldots \ldots \ldots \ldots$ & $2.95 \pm 0.01$ & $\ldots$ & 0.87 & $2.92 \pm 0.18$ & $0.59 \pm 0.11$ & 0.87 & $2.07 \pm 0.03$ & $0.66 \pm 0.08$ & 0.88 \\
\hline $27 \ldots \ldots \ldots \ldots$ & $2.15 \pm 0.21$ & $0.87 \pm 0.32$ & 0.87 & $2.15 \pm 0.17$ & $1.13 \pm 0.19$ & 0.91 & $2.32 \pm 0.07$ & $0.36 \pm 0.11$ & 0.87 \\
\hline $28 \ldots \ldots \ldots \ldots . . .$. & $3.26 \pm 0.17$ & $0.63 \pm 0.24$ & 0.78 & $3.35 \pm 0.18$ & $0.75 \pm 0.17$ & 0.88 & $3.42 \pm 0.23$ & & 0.84 \\
\hline $29 \ldots \ldots \ldots \ldots \ldots$ & $2.32 \pm 0.2$ & & 0.88 & $2.09 \pm 0.02$ & $3.82 \pm 2.64$ & 0.9 & $1.83 \pm 0.08$ & $1.11 \pm 0.16$ & 0.89 \\
\hline 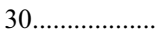 & $2.01 \pm 0.08$ & $1.21 \pm 0.36$ & 0.86 & $2.07 \pm 0.1$ & $1.73 \pm 0.22$ & 0.93 & $2.31 \pm 0$ & $0.4 \pm 0.07$ & 0.89 \\
\hline $31 \ldots \ldots \ldots$ & $1.74 \pm 0.08$ & $\ldots$ & 0.93 & $1.79 \pm 0.08$ & $\ldots$ & 0.95 & $2.05 \pm 0.23$ & $1.21 \pm 0.43$ & 0.89 \\
\hline $32 \ldots \ldots \ldots \ldots$ & $2.75 \pm 0.26$ & $\ldots$ & 0.87 & $2.88 \pm 0.12$ & $\ldots$ & 0.92 & $3.03 \pm 0.11$ & $\ldots$ & 0.91 \\
\hline 33.................... & $3.1 \pm 0.67$ & $\ldots$ & 0.69 & $3.1 \pm 0.25$ & $\ldots$ & 0.8 & $3.01 \pm 0.08$ & $\ldots$ & 0.88 \\
\hline $34 \ldots \ldots \ldots \ldots \ldots \ldots$ & $1.74 \pm 0.31$ & $0.44 \pm 0.22$ & 0.79 & $1.7 \pm 0.16$ & $0.46 \pm 0.08$ & 0.89 & $2.76 \pm 0.28$ & $0.41 \pm 0.21$ & 0.79 \\
\hline $35 \ldots \ldots \ldots \ldots \ldots$ & $2.46 \pm 0.13$ & $1.57 \pm 0.72$ & 0.88 & $2.56 \pm 0.15$ & & 0.88 & $2.79 \pm 0.09$ & & 0.89 \\
\hline 36.................... & $1.53 \pm 0.02$ & $0.93 \pm 0.13$ & 0.9 & $1.64 \pm 0.08$ & $0.68 \pm 0.03$ & 0.94 & $2.32 \pm 0.1$ & $0.34 \pm 0.01$ & 0.86 \\
\hline 37..................... & $3.69 \pm 0.25$ & $\ldots$ & 0.73 & $3.24 \pm 0.1$ & $\ldots$ & 0.86 & $2.25 \pm 0.13$ & $\ldots$ & 0.79 \\
\hline 38................... & $2.86 \pm 0.01$ & $0.95 \pm 0.18$ & 0.9 & $2.79 \pm 0.06$ & $1.45 \pm 0.31$ & 0.89 & $2.75 \pm 0.18$ & $1.52 \pm 0.58$ & 0.89 \\
\hline 39................ & $2.01 \pm 0.12$ & $\ldots$ & 0.88 & $1.9 \pm 0.08$ & $1.04 \pm 0.08$ & 0.92 & $1.61 \pm 0.09$ & $0.28 \pm 0.06$ & 0.86 \\
\hline $40 \ldots$ & $1.88 \pm 0.05$ & $0.62 \pm 0.12$ & 0.88 & $1.77 \pm 0.16$ & $1.34 \pm 0.3$ & 0.9 & $1.83 \pm 0.12$ & $0.32 \pm 0.1$ & 0.87 \\
\hline $41 \ldots \ldots \ldots$ & $1.59 \pm 0.11$ & $2.8 \pm 1.52$ & 0.92 & $1.7 \pm 0.01$ & $5.54 \pm 1.97$ & 0.93 & $1.88 \pm 0.13$ & $0.69 \pm 0.2$ & 0.89 \\
\hline $42 \ldots \ldots \ldots \ldots$ & $1.92 \pm 0.03$ & $2.01 \pm 0.34$ & 0.9 & $1.98 \pm 0.09$ & $4.24 \pm 1.15$ & 0.93 & $2.17 \pm 0.06$ & $0.74 \pm 0.19$ & 0.88 \\
\hline $43 \ldots \ldots \ldots \ldots$ & $1.98 \pm 0.04$ & $1.68 \pm 0.11$ & 1.03 & $1.91 \pm 0.03$ & $2.57 \pm 0.37$ & 0.92 & $2.0 \pm 0.04$ & $0.41 \pm 0.07$ & 0.9 \\
\hline 44.................... & $1.34 \pm 0.03$ & $8.63 \pm 3.11$ & 0.93 & $1.33 \pm 0.07$ & $10.0 \pm 2.49$ & 0.94 & $1.49 \pm 0.06$ & $1.37 \pm 0.4$ & 0.89 \\
\hline $45 \ldots \ldots \ldots \ldots \ldots$ & $2.25 \pm 0.04$ & $3.22 \pm 1.47$ & 0.87 & $2.02 \pm 0.09$ & $1.72 \pm 0.3$ & 0.9 & $2.25 \pm 0.1$ & $0.85 \pm 0.11$ & 0.91 \\
\hline $46 \ldots \ldots \ldots \ldots$ & $1.6 \pm 0.01$ & $3.26 \pm 1.23$ & 0.91 & $1.52 \pm 0.08$ & $8.17 \pm 3.37$ & 0.94 & $1.5 \pm 0.04$ & $1.27 \pm 0.15$ & 0.9 \\
\hline 47.................... & $1.77 \pm 0.19$ & $0.87 \pm 0.13$ & 0.89 & $1.71 \pm 0.13$ & $1.26 \pm 0.2$ & 0.92 & $2.02 \pm 0.1$ & $0.41 \pm 0.02$ & 0.91 \\
\hline $48 \ldots \ldots \ldots \ldots . . .$. & $1.64 \pm 0.05$ & $0.69 \pm 0.09$ & 0.87 & $1.59 \pm 0.11$ & $0.71 \pm 0.06$ & 0.91 & $1.96 \pm 0.17$ & $0.35 \pm 0.06$ & 0.88 \\
\hline 49................... & $1.93 \pm 0.29$ & $4.89 \pm 2.44$ & 0.91 & $1.87 \pm 0.11$ & $4.64 \pm 1.14$ & 0.93 & $2.17 \pm 0.12$ & $4.34 \pm 3.1$ & 0.88 \\
\hline $50 \ldots \ldots \ldots \ldots$ & $1.35 \pm 0.27$ & $1.53 \pm 0.37$ & 0.9 & $1.3 \pm 0.13$ & $1.99 \pm 0.41$ & 0.93 & $1.98 \pm 0.11$ & $0.74 \pm 0.24$ & 0.9 \\
\hline $51 \ldots \ldots \ldots \ldots \ldots$ & $2.58 \pm 0.05$ & $\ldots$ & 0.89 & $2.64 \pm 0.1$ & $3.04 \pm 0.97$ & 0.91 & $2.85 \pm 0.08$ & $0.65 \pm 0.13$ & 0.89 \\
\hline $52 \ldots \ldots \ldots \ldots \ldots$ & $2.3 \pm 0.05$ & $\ldots$ & 0.89 & $2.27 \pm 0.08$ & & 0.91 & $2.63 \pm 0.07$ & & 0.91 \\
\hline $53 \ldots \ldots \ldots \ldots$ & $2.07 \pm 0.12$ & $0.46 \pm 0.02$ & 0.87 & $2.07 \pm 0.12$ & $0.68 \pm 0.09$ & 0.9 & $2.36 \pm 0.14$ & $0.33 \pm 0$ & 0.87 \\
\hline $54 \ldots \ldots \ldots \ldots \ldots . .$. & $2.05 \pm 0.13$ & $1.04 \pm 0.17$ & 0.89 & $2.04 \pm 0.09$ & $2.02 \pm 0.41$ & 0.92 & $2.47 \pm 0.14$ & $1.17 \pm 0.33$ & 0.89 \\
\hline $55 \ldots \ldots \ldots \ldots \ldots$ & $2.16 \pm 0.16$ & $1.19 \pm 0.23$ & 0.9 & $2.09 \pm 0.1$ & $1.31 \pm 0.14$ & 0.93 & $2.37 \pm 0.12$ & $0.47 \pm 0.09$ & 0.88 \\
\hline $56 \ldots \ldots \ldots \ldots \ldots \ldots$ & $1.94 \pm 0.11$ & $1.56 \pm 0.44$ & 0.9 & $1.93 \pm 0.16$ & $2.03 \pm 0.36$ & 0.92 & $2.28 \pm 0.1$ & $\ldots$ & 0.88 \\
\hline 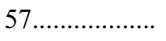 & $1.75 \pm 0.18$ & $0.77 \pm 0.05$ & 0.9 & $1.6 \pm 0.09$ & $1.39 \pm 0.22$ & 0.93 & $2.18 \pm 0.14$ & $0.79 \pm 0.22$ & 0.9 \\
\hline $58 \ldots \ldots \ldots \ldots \ldots . .$. & $1.41 \pm 0.27$ & $0.61 \pm 0.1$ & 0.88 & $1.36 \pm 0.18$ & $0.66 \pm 0.07$ & 0.91 & $2.4 \pm 0.2$ & $0.84 \pm 0.18$ & 0.89 \\
\hline $59 \ldots \ldots \ldots \ldots$ & $1.25 \pm 0.02$ & $4.12 \pm 0.62$ & 0.93 & $1.22 \pm 0.06$ & $5.98 \pm 0.7$ & 0.95 & $1.57 \pm 0.05$ & & 0.95 \\
\hline $60 \ldots \ldots \ldots \ldots \ldots$ & $1.52 \pm 0.07$ & $1.23 \pm 0.06$ & 0.95 & $1.43 \pm 0.06$ & $1.37 \pm 0.12$ & 0.93 & $1.49 \pm 0.05$ & $0.5 \pm 0.07$ & 0.9 \\
\hline 61.................... & $0.99 \pm 0.08$ & $3.82 \pm 0.33$ & 0.92 & $1.11 \pm 0.04$ & $3.54 \pm 0.4$ & 0.94 & $1.14 \pm 0.07$ & $2.6 \pm 0.67$ & 0.88 \\
\hline $62 \ldots \ldots \ldots \ldots \ldots$ & $1.96 \pm 0.02$ & $1.13 \pm 0.11$ & 0.95 & $1.81 \pm 0.09$ & $2.07 \pm 0.41$ & 0.92 & $2.15 \pm 0.17$ & $2.04 \pm 1.55$ & 0.89 \\
\hline 63.................. & $2.76 \pm 0.01$ & .. & 0.9 & $2.49 \pm 0.03$ & & 0.92 & $2.18 \pm 0.15$ & & 0.89 \\
\hline
\end{tabular}


TABLE 2 - Continued

\begin{tabular}{|c|c|c|c|c|c|c|c|c|c|}
\hline \multirow[b]{2}{*}{ Number } & \multicolumn{3}{|c|}{ CARBON } & \multicolumn{3}{|c|}{ OXYGEN } & \multicolumn{3}{|c|}{ IRON } \\
\hline & $\gamma^{\mathrm{a}}$ & $E_{0}^{\mathrm{b}, \mathrm{c}}$ & $\chi_{v}^{2}$ & $\gamma^{\mathrm{a}}$ & $E_{0}^{\mathrm{b}, \mathrm{c}}$ & $\chi_{v}^{2}$ & $\gamma^{\mathrm{a}}$ & $E_{0}^{\mathrm{b}, \mathrm{c}}$ & $\chi_{v}^{2}$ \\
\hline $64 \ldots \ldots \ldots \ldots . .$. & $1.09 \pm 0.19$ & $0.64 \pm 0.09$ & 0.88 & $1.02 \pm 0.11$ & $0.67 \pm 0.08$ & 0.9 & $1.79 \pm 0.18$ & $0.28 \pm 0.04$ & 0.87 \\
\hline $65 \ldots \ldots \ldots$ & $2.61 \pm 0.02$ & $3.24 \pm 1.76$ & 0.89 & $2.46 \pm 0.06$ & $1.61 \pm 0.45$ & 0.9 & $2.34 \pm 0.06$ & $0.94 \pm 0.48$ & 0.88 \\
\hline 66.................... & $1.45 \pm 0.11$ & $0.68 \pm 0.21$ & 0.86 & $1.43 \pm 0.12$ & $2.15 \pm 1.45$ & 0.91 & $2.36 \pm 0.25$ & $1.16 \pm 0.42$ & 0.89 \\
\hline 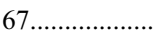 & $1.83 \pm 0.33$ & $1.33 \pm 0.22$ & 0.91 & $1.56 \pm 0.1$ & $1.23 \pm 0.13$ & 0.92 & $1.9 \pm 0.14$ & $0.66 \pm 0.18$ & 0.9 \\
\hline $68 \ldots \ldots \ldots \ldots$ & $2.28 \pm 0.21$ & $0.37 \pm 0.1$ & 0.78 & $2.17 \pm 0.1$ & $0.4 \pm 0.02$ & 0.93 & $2.63 \pm 0.19$ & $0.25 \pm 0.04$ & 0.79 \\
\hline $69 \ldots \ldots \ldots$ & $1.08 \pm 0.24$ & $2.01 \pm 0.38$ & 0.92 & $0.9 \pm 0.1$ & $1.19 \pm 0.06$ & 0.93 & $0.8 \pm 0.09$ & $0.41 \pm 0.04$ & 0.88 \\
\hline 70....................... & $1.44 \pm 0.2$ & $1.64 \pm 0.31$ & 0.9 & $1.16 \pm 0.04$ & $1.25 \pm 0.1$ & 0.92 & $1.29 \pm 0.1$ & $0.42 \pm 0.07$ & 0.89 \\
\hline 71...................... & $1.59 \pm 0.08$ & $1.99 \pm 0.41$ & 0.91 & $1.49 \pm 0.08$ & $3.57 \pm 0.78$ & 0.92 & $1.8 \pm 0.18$ & $0.56 \pm 0.12$ & 0.89 \\
\hline 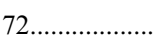 & $2.2 \pm 0.07$ & $0.85 \pm 0.34$ & 0.78 & $2.22 \pm 0.05$ & $1.55 \pm 0.42$ & 0.87 & $2.08 \pm 0.28$ & $1.55 \pm 0.56$ & 0.88 \\
\hline
\end{tabular}

Note.-See Table 1 of Desai et al. (2003) and Table A1 of the Appendix for a list of shock arrival times at $A C E$.

${ }^{\text {a }}$ Spectral index $\gamma$ is estimated from fitting $j=j_{0} E^{-\gamma}$ to the $0.1-0.5 \mathrm{MeV}$ nucleon ${ }^{-1}$ ULEIS intensities.

${ }^{\mathrm{b}}$ Units of $e$-folding energy $E_{0}$ are $\mathrm{MeV}$ nucleon ${ }^{-1} . E_{0}$ is obtained by fitting the $0.1-5.0 \mathrm{MeV}$ nucleon ${ }^{-1}$ ULEIS intensities by $j=j_{0} E^{-\gamma} \exp \left(-E / E_{0}\right)$.

${ }^{c}$ No values are provided for events lacking spectral breaks between 0.1 and $5.0 \mathrm{MeV}$ nucleon $^{-1}$.

decrease in $\mathrm{Fe} / \mathrm{O}$ with increasing energy shown in Figure $3 d$. In contrast, the values of $E_{0}$ for $\mathrm{C}, \mathrm{O}$, and $\mathrm{Fe}$ in event 18 were similar, so all the ratios were constant over the energy range. Finally, for event 37 the Fe spectrum was significantly harder than the $\mathrm{C}$ and $\mathrm{O}$ spectra, leading to a factor of $\sim 8$ increase in $\mathrm{Fe} / \mathrm{O}$ over the energy range.

\subsection{Shock-associated Spectra Measured by ULEIS and SIS}

Figure 4 shows $(a) \mathrm{C}, \mathrm{O}$, and $\mathrm{Fe}$ fluences and $(b) \mathrm{C} / \mathrm{O}$ and $\mathrm{Fe} / \mathrm{O}$ ratios versus energy for event 61 . The ULEIS fluences and abundances are obtained from November 232232 UT to November 242213 UT, while those from SIS are obtained from November 231909 UT to November 241951 UT. Note the excellent agreement between the $\mathrm{O}$ fluences at the point where the two instruments overlap in energy. For this event, the Jones \& Ellison expression provided reasonable visual fits for the $\mathrm{C}$ and $\mathrm{O}$ spectra, but clearly not for the Fe spectrum. Figure $4 a$ shows that the spectra for all three species rolled over around $10 \mathrm{MeV}$ nucleon $^{-1}$, and above $\sim 10 \mathrm{MeV}$ nucleon $^{-1}$ the $\mathrm{Fe}$ spectrum became steeper than those of $\mathrm{C}$ and $\mathrm{O}$. Consequently, the $\mathrm{C} / \mathrm{O}$ ratio in Figure $4 b$ remained relatively constant up to $\sim 50 \mathrm{MeV}$ nucleon $^{-1}$, while the $\mathrm{Fe} / \mathrm{O}$ ratio was constant at $\sim 0.4$ up to $\sim 2 \mathrm{MeV}$ nucleon ${ }^{-1}$ and then decreased by about a factor of 5 above $\sim 12 \mathrm{MeV}$ nucleon $^{-1}$ to $\sim 0.09$. In summary, the Fe spectrum in event 61 rolled over at a lower energy than the $\mathrm{C}$ and $\mathrm{O}$ spectra, and this effect occurred between $\sim 2-20 \mathrm{MeV}$ nucleon $^{-1}$, i.e., above the energy range of the ULEIS measurements.

\subsection{Properties of Spectral Fit Parameters $\gamma$ and $E_{0}$}

Figure 5 investigates the relationship between the 0.1$0.5 \mathrm{MeV}$ nucleon ${ }^{-1}$ spectral indices $\gamma$ of $\mathrm{C}, \mathrm{O}$, and Fe nuclei listed in Table 2. Note that the values of $\gamma$ fell in the range $\sim 1-3.5$. In general, the $\mathrm{C}$ and $\mathrm{Fe}$ spectral indices were well correlated with those of $\mathrm{O}$. Although the correlation between the $\gamma$-values of $\mathrm{Fe}$ and $\mathrm{O}\left(r^{2} \sim 0.62\right)$ was smaller than that between the $\gamma$-values of $\mathrm{C}$ and $\mathrm{O}\left(r^{2} \sim 0.96\right)$, it was nevertheless highly significant.

Figure 6 shows scatter plots of the $e$-folding energy $E_{0}$ for $\mathrm{O}$ versus $(a) \theta_{B n}$ and (b) $V_{S}$. The figure clearly shows that $E_{0}$ was poorly correlated with both shock parameters. We also found (not shown) that $\gamma$ and $E_{0}$ for all species were poorly correlated with various other shock strength parameters, such as the
Mach number $M_{A}$, the magnetic $M$ and density $H$ compression ratios, and the shock speed $V_{\mathrm{IP}}$.

\section{RELATIVE BEHAVIOR OF O AND Fe SPECTRA}

\subsection{Energy Dependence of $\mathrm{Fe} / \mathrm{O}$}

In order to survey the relative behavior of $\mathrm{O}$ and $\mathrm{Fe}$ spectra between $\sim 0.1$ and $100 \mathrm{MeV}$ nucleon ${ }^{-1}$ at the 72 IP shocks, we used the energy dependence of the $\mathrm{Fe} / \mathrm{O}$ ratios, such as those shown in the right-hand panels of Figures 3 and 4. For each event, we calculated the average ULEIS $\mathrm{Fe} / \mathrm{O}$ ratios in three different energy ranges, namely, $0.11-0.32,0.32-0.91$, and $0.91-2.56 \mathrm{MeV}$ nucleon ${ }^{-1}$; hereafter these are referred to as $0.22,0.62$, and $1.74 \mathrm{MeV}^{\text {nucleon }}{ }^{-1}$, respectively. For the ULEIS $\mathrm{Fe} / \mathrm{O}$ ratio calculations, we required that there were finite $\mathrm{Fe} / \mathrm{O}$ measurements with a relative uncertainty of less than $35 \%$ in each of three logarithmically spaced bins within the above three energy ranges. The SIS Fe/O ratios cover the range $12-60 \mathrm{MeV}$ nucleon $^{-1}$.

Figure 7 shows scatter plots of $0.22 \mathrm{MeV}$ nucleon $^{-1} \mathrm{Fe} / \mathrm{O}$ ratios versus those measured in the three higher energy intervals. Events 1, 5, 9, and 19 are excluded from Figure $7 a$ because of insufficient counting statistics for Fe. Each panel shows a diagonal line representing equal $\mathrm{Fe} / \mathrm{O}$ ratios at both energies plotted and dotted lines enclosing a band corresponding to deviations of \pm 0.33 from unity. In each panel, events are color coded according to whether they lie above the band (red), within it (green), or below it (blue), corresponding respectively to $\mathrm{Fe} / \mathrm{O}$ increasing, constant, or decreasing with energy.

Several important features are evident from the figure. First, the majority of the events have $\mathrm{Fe} / \mathrm{O}$ ratios that either decrease or remain constant with energy, while about $\sim 7 \%$ of the events have $\mathrm{Fe} / \mathrm{O}$ that increase with energy. Second, the occurrence frequency of events with decreasing $\mathrm{Fe} / \mathrm{O}$ ratios is substantially larger at higher energies. Third, events where $\mathrm{Fe} / \mathrm{O}$ decreases with energy have a significantly lower $\mathrm{Fe}$ abundance at higher energy when compared with that measured at $0.22 \mathrm{MeV}^{\text {nucleon }}{ }^{-1}$.

The main statistical results regarding the energy dependence of $\mathrm{Fe} / \mathrm{O}$ during the IP shock-associated events are summarized in Table 3. The table shows that Fe/O generally decreased with energy for the events that produced the largest intensities at SIS energies. We also performed a similar 

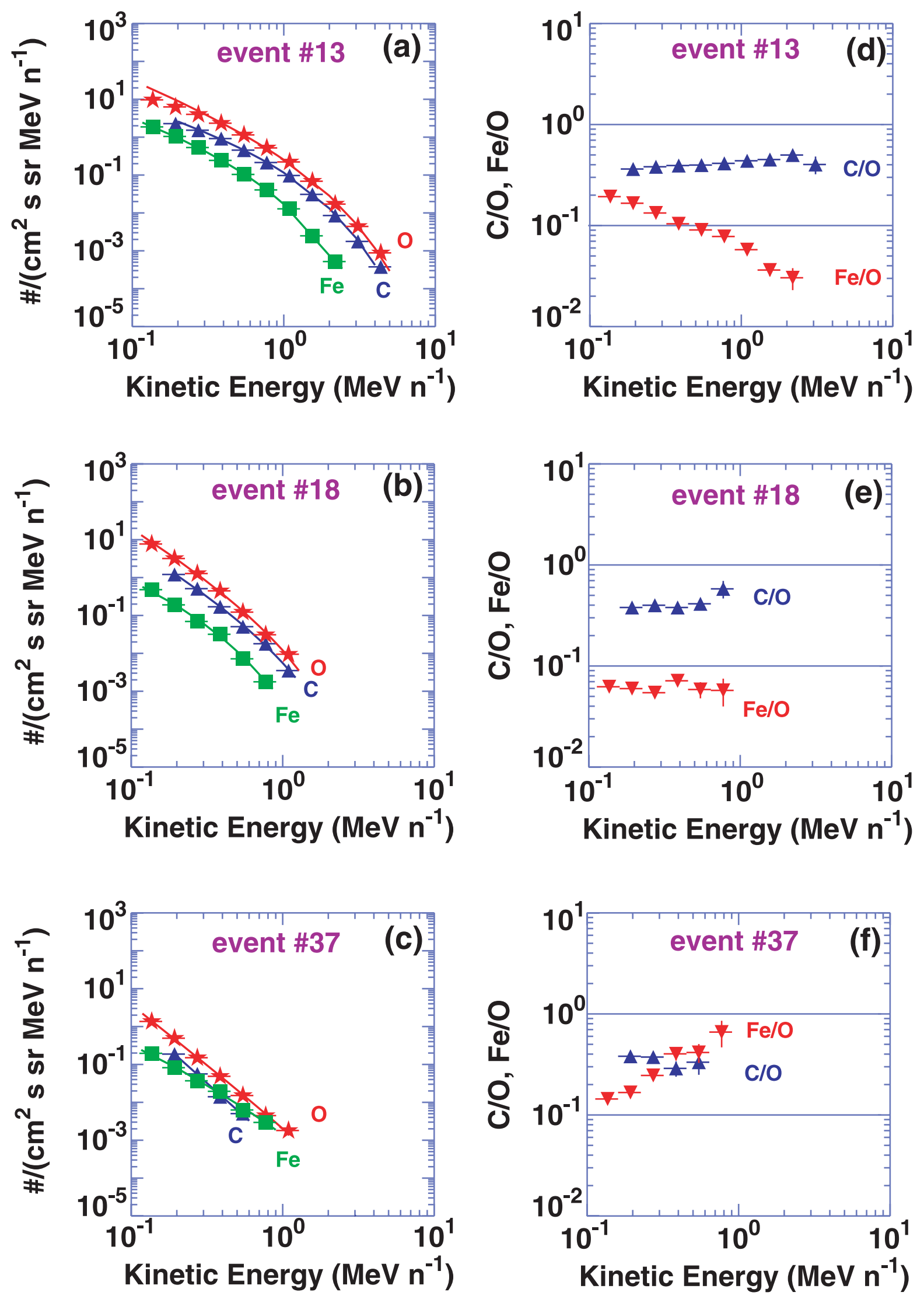

Fig. 3.-Left panels: Energy spectra of C, O, and Fe during events 13, 18, and 37. The solid curves show fits with the Jones \& Ellison expression (see text for details). Right panels: $\mathrm{C} / \mathrm{O}$ and $\mathrm{Fe} / \mathrm{O}$ ratios vs. energy for the three IP shock events. 

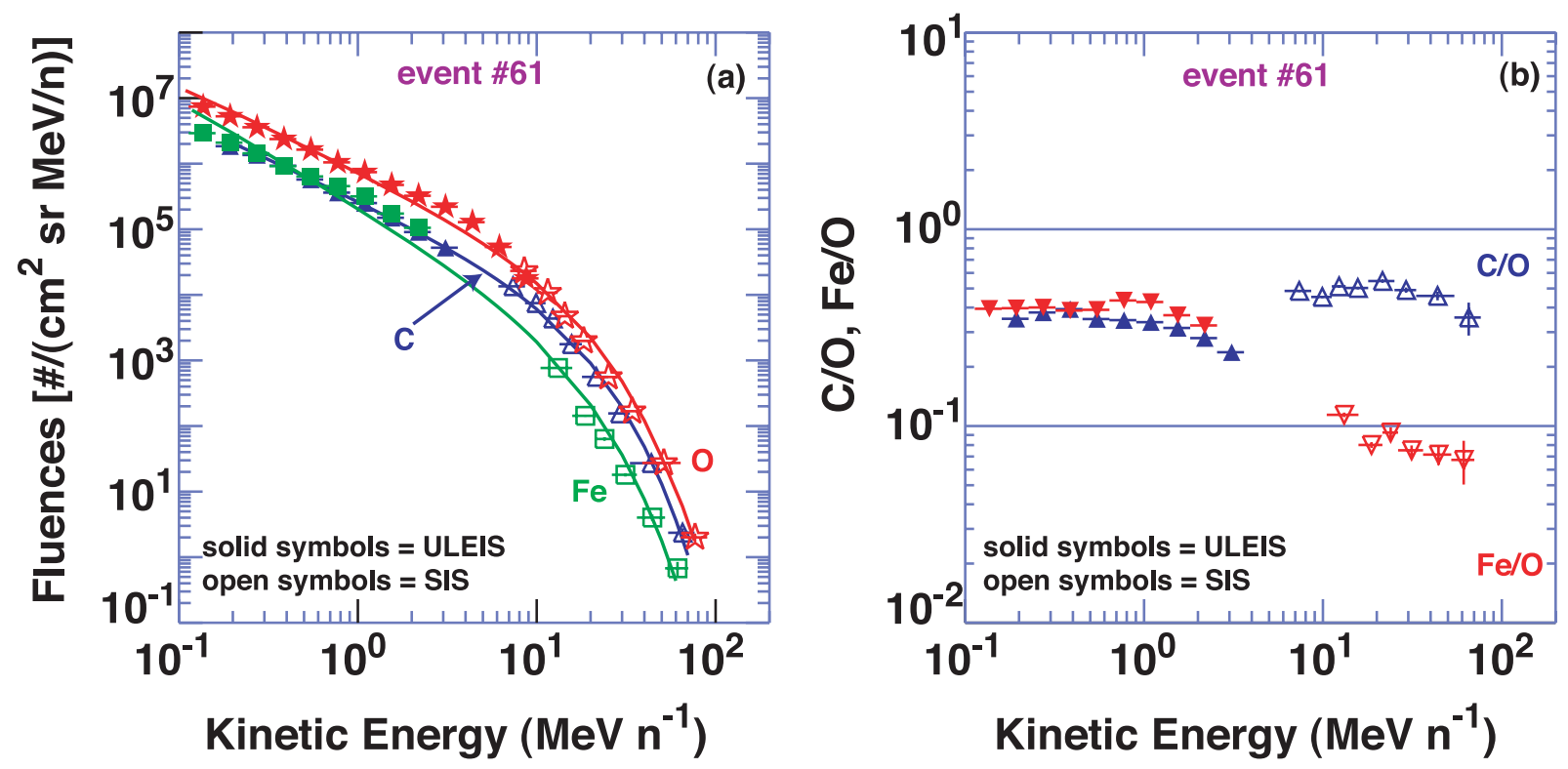

Fig. 4. - (a) $\mathrm{C}, \mathrm{O}$, and Fe fluences and $(b) \mathrm{C} / \mathrm{O}$ and Fe/O ratios vs. energy for event 61 in Fig. 2. The solid curves in $(a)$ show fits with the Jones \& Ellison expression.

analysis to investigate the relative behavior of $\mathrm{C}$ and $\mathrm{O}$ spectra and found that the $\mathrm{C} / \mathrm{O}$ ratio remained relatively constant with energy (see Figs. 3 and 4) for all the 72 events surveyed here.

\subsection{Properties of Energy-dependent Parameter $\Gamma_{\mathrm{Fe}}$}

To investigate the relationship between the energy dependence of $\mathrm{Fe} / \mathrm{O}$ and other properties of the IP shocks, we define an energy-dependence parameter $\Gamma_{\mathrm{Fe}}$ as the ratio of the mean $\mathrm{Fe} / \mathrm{O}$ at $0.62 \mathrm{MeV}$ nucleon $^{-1}$ divided by the mean $\mathrm{Fe} / \mathrm{O}$ at $0.22 \mathrm{MeV}$ nucleon $^{-1}$. This is equivalent to the perpendicular distance from the 1:1 line in Figure $7 a$. Figure 8 plots $\Gamma_{\mathrm{Fe}}$ versus $(a)$ the $0.1-0.5 \mathrm{MeV}$ nucleon ${ }^{-1} \mathrm{O}$ spectral index and (b) the $0.5-2.0 \mathrm{MeV}$ nucleon ${ }^{-1} \mathrm{O}$ fluence. The figure clearly shows that $\Gamma_{\mathrm{Fe}}$ was positively correlated with the spectral index and negatively correlated with the fluence. In particular, events with $\Gamma_{\mathrm{Fe}}>1.33$ tended to have low $\mathrm{O}$ fluences, less than $10^{3}$ particles $\left(\mathrm{cm}^{2} \mathrm{sr} \mathrm{MeV} \text { nucleon }{ }^{-1}\right)^{-1}$, and soft $\mathrm{O}$ spectra $(\gamma>2)$; i.e., these were some of the weakest particle events in our survey. Hereafter we refer to "weak" events as those with $\mathrm{O}$ fluence less than $10^{3}$ particles $\left(\mathrm{cm}^{2} \mathrm{sr} \mathrm{MeV}\right.$ nucleon $\left.^{-1}\right)^{-1}$ and $\mathrm{O}$ spectral index greater than 2. The remaining events are referred to as "strong" events.

Figure 9 shows (a) $\Gamma_{\mathrm{Fe}}$ versus the 0.5-2.0 $\mathrm{MeV}$ nucleon ${ }^{-13} \mathrm{He} /{ }^{4} \mathrm{He}$ ratio and $(b)$ the ${ }^{3} \mathrm{He} /{ }^{4} \mathrm{He}$ ratio versus $\theta_{B n}$ for 43 of the $45^{3} \mathrm{He}$-rich IP shocks identified in Desai et al. (2003). Figure $9 a$ shows that although $\Gamma_{\mathrm{Fe}}$ was positively correlated
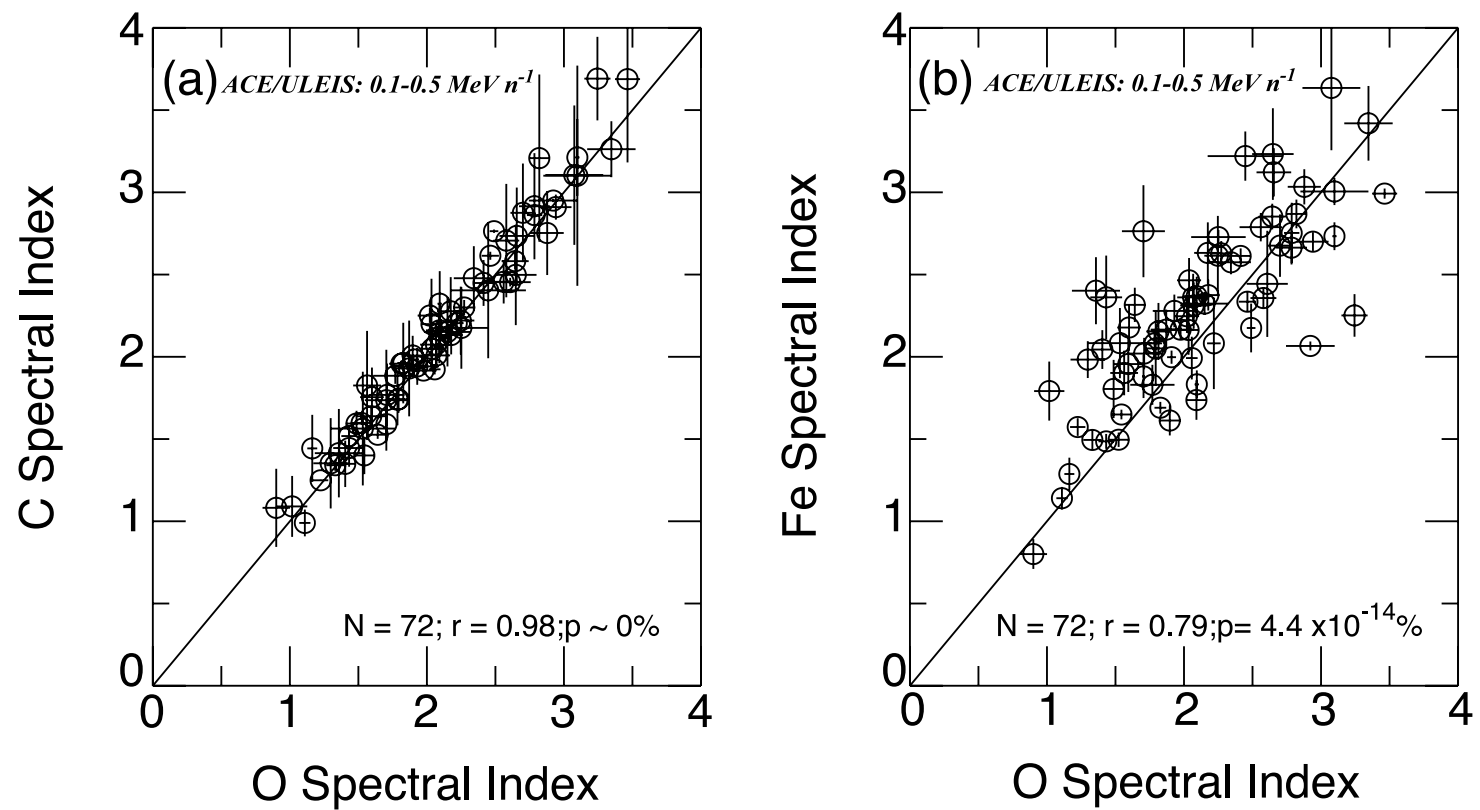

FIG. 5.-Low-energy power-law spectral indices of $\mathrm{C}$ and Fe vs. that of $\mathrm{O}$ (see Table 2 ). The solid line has slope $=1$ and is drawn through $[1,1] ;, N, r$, and $p$ denote the number of points, the linear correlation coefficient, and its statistical significance. 

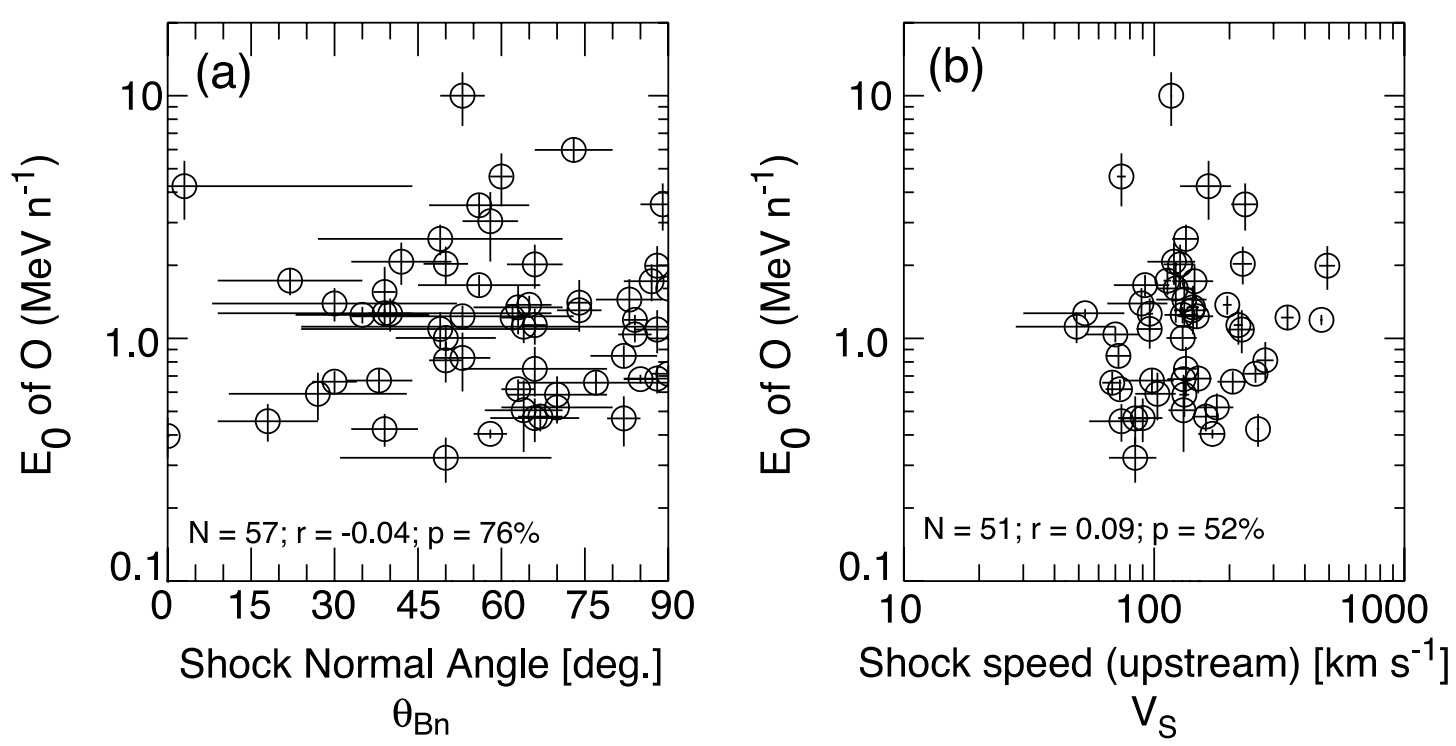

Fig. 6. - The $e$-folding energy $E_{0}$ of $\mathrm{O}$ vs. (a) $\theta_{B n}$ and (b) $V_{S}$.

with the ${ }^{3} \mathrm{He} /{ }^{4} \mathrm{He}$ ratio, only 4 of the 13 events with a ${ }^{3} \mathrm{He} /{ }^{4} \mathrm{He}$ ratio greater than $2 \%$ also exhibited an increase in $\mathrm{Fe} / \mathrm{O}$ with energy. Figure $9 b$ shows that the ${ }^{3} \mathrm{He} /{ }^{4} \mathrm{He}$ ratio was uncorrelated with $\theta_{B n}$ and further that IP shocks with $\theta_{B n} \geq 60^{\circ}$ had a wide range of ${ }^{3} \mathrm{He} /{ }^{4} \mathrm{He}$ ratios with values between $\sim 2 \times 10^{-3}$ and $2 \times 10^{-1}$. We also found (not shown) that $\Gamma_{\mathrm{Fe}}$ was poorly correlated with parameters that characterize the strength of the IP shocks at 1 AU.

\section{RELATION BETWEEN SHOCK-ASSOCIATED AND AMBIENT SUPRATHERMAL IONS}

\subsection{Fractionation Pattern}

In order to investigate the fractionation of elemental abundances in IP shocks, we plot in Figure $10 a$ the $0.32-0.45 \mathrm{MeV}$ nucleon $^{-1}$ abundance ratios relative to $\mathrm{O}$ versus the $\mathrm{Fe} / \mathrm{C}$ ratio measured during the 72 shock events in the survey. The dashed lines are given by (e.g., Breneman \& Stone 1985)

$$
\left(\frac{\mathrm{X}}{\mathrm{O}}\right)_{\mathrm{IP}}=\left(\frac{\mathrm{X}}{\mathrm{O}}\right)_{\mathrm{GSEPS}}\left(\frac{M_{\mathrm{X}} / Q_{\mathrm{X}}}{M_{\mathrm{O}} / Q_{\mathrm{O}}}\right)^{\delta},
$$

where $(\mathrm{X} / \mathrm{O})_{\mathrm{IP}}$ is the IP shock abundance of element $\mathrm{X}$ relative to $\mathrm{O},(\mathrm{X} / \mathrm{O})_{\mathrm{GSEPs}}$ is the average abundance ratio measured in gradual SEP events between 5 and $12 \mathrm{MeV}$ nucleon ${ }^{-1}$ (from Reames 1995), $M_{\mathrm{X}} / Q_{\mathrm{X}}$ and $M_{\mathrm{O}} / Q_{\mathrm{O}}$ are the mass-to-charge ratios of element $\mathrm{X}$ and $\mathrm{O}$, respectively, and $\delta$ is the power-law index with values for Figure 10 ranging from -1.5 to 3.5. Note that $\delta>0$ and $\delta<0$ respectively represent enhancements and depletions when compared with the corresponding gradual SEP value. The charge states for various species are taken as the mean ionization states measured in gradual SEP events between 0.18 and $0.44 \mathrm{MeV}$ nucleon ${ }^{-1}$, namely, $\mathrm{He}^{2+}$, $\mathrm{C}^{5.6+}, \mathrm{N}^{6.6+}, \mathrm{O}^{6.8+}, \mathrm{Ne}^{8.2+}, \mathrm{Mg}^{8.9+}, \mathrm{Si}^{9.5+}, \mathrm{S}^{10.2+}, \mathrm{Ca}^{10.8+}$, and $\mathrm{Fe}^{11.6+}$ (Klecker et al. 1999; Möbius et al. 1999, 2000). Although cases of significant changes in the charge state of $\mathrm{Fe}$ with energy during some large SEP events have been reported, we note that the biggest changes were observed in the range of tens of $\mathrm{MeV}$ nucleon ${ }^{-1}$, i.e., at the very highest energy portion of the SIS instrument (e.g., Leske et al. 1999). In association with the passage of IP shocks at $1 \mathrm{AU}$, however, the mean $\mathrm{Fe}$ charge state has been observed to exhibit relatively modest ( $\sim 1$ charge unit) increases with energy between $\sim 0.1$ and
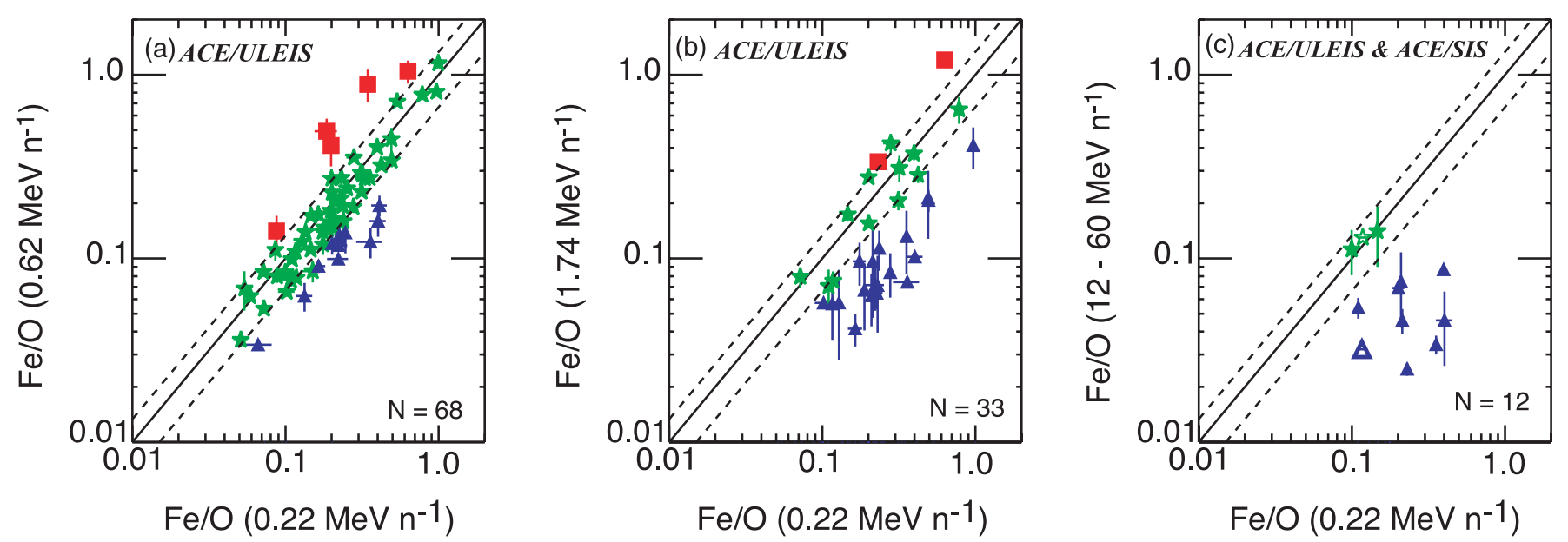

FIG. 7.-Energy-dependent behavior of Fe/O as measured by ULEIS and SIS between 0.1 and $60 \mathrm{MeV}^{\text {nucleon }}{ }^{-1}$ (see text for details). The dashed lines identify

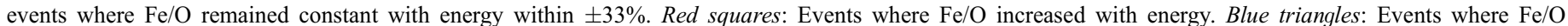
decreased with energy. Green stars: Events where Fe/O remained constant with energy. The open symbols in (c) denote the upper limit events for SIS. 
TABLE 3

Number of Events versus Qualitative Behavior of Fe/O at Higher Energies Compared with Fe/O at $0.22 \mathrm{MeV}^{N_{u}}$ legon $^{-1}$

\begin{tabular}{|c|c|c|c|c|c|c|}
\hline Behavior Compared with $0.22 \mathrm{MeV}$ Nucleon $^{-1}$ & \multicolumn{2}{|c|}{$0.62 \mathrm{MeV}$ NUCLEON $^{-1}$ (ULEIS) } & \multicolumn{2}{|c|}{$1.74 \mathrm{MeV}$ NuCLEON $^{-1}$ (ULEIS) } & \multicolumn{2}{|c|}{$12-60 \mathrm{MeV}$ NuCLEON $^{-1}$ (SIS) } \\
\hline$\ldots$ & 5 & $7.4 \pm 3.2$ & 2 & $6.1 \pm 4.2$ & 0 & 0.00 \\
\hline $\mathrm{Fe} / \mathrm{O}$ constant & 51 & $75.0 \pm 5.3$ & 12 & $36.4 \pm 8.4$ & 3 & $25.0 \pm 12.5$ \\
\hline 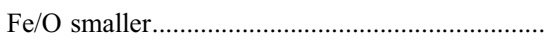 & 12 & $17.7 \pm 4.6$ & 19 & $57.6 \pm 8.6$ & 9 & $75.0 \pm 12.5$ \\
\hline Total, $N$ & 68 & $\ldots$ & 33 & $\ldots$ & 12 & $\ldots$ \\
\hline
\end{tabular}

NoтE.-Occurrence frequencies $p$ and their uncertainties are estimated using the mean $\mu=N p$ and standard deviation $\sigma=[N p(1-p)]^{1 / 2}$ of the multinomial distribution.

2.0 MeV nucleon ${ }^{-1}$ (e.g., Klecker et al. 2003). For the dashed lines, $\chi_{v}^{2}=0.67$ with probability $P \sim 72 \%$, which is an excellent representation of the data (from Bevington \& Robinson 1992). In contrast, similar fits using the average solar wind abundances and ionization states from von Steiger et al. (1997, 2000) yielded $\chi_{v}^{2}=2.24, P \sim 2 \%$ for the fast solar wind and $\chi_{v}^{2}=3.15, P \sim 1.4 \%$ for the slow solar wind, indicating poor representation of the IP shock abundances (also see Desai et al. 2003).

Figure $10 \mathrm{~b}$ displays the ambient suprathermal abundances in the same fashion as Figure 10a. Note that both the slope and normalization constant for each element in IP shocks are remarkably similar to those of the ambient suprathermals. The values of $\chi_{v}^{2}$ and $P$ for the three reference populations are $\chi_{v}^{2}=1.15, P \sim 33 \%$ for gradual SEP events; $\chi_{v}^{2}=2.21$, $P \sim 2.4 \%$ for the fast solar wind; and $\chi_{v}^{2}=2.61, P \sim 0.8 \%$ for the slow solar wind. Thus, equation (1) provides very good fits to both the IP shock and the ambient suprathermal ion abundances when compared with gradual SEP abundances but not when compared with solar wind values.

\subsection{Energy Dependence of the Abundances}

Figure 11 displays the $\mathrm{C} / \mathrm{O}$ and $\mathrm{Fe} / \mathrm{O}$ ratios versus energy for the three representative IP shocks shown in Figure 3,

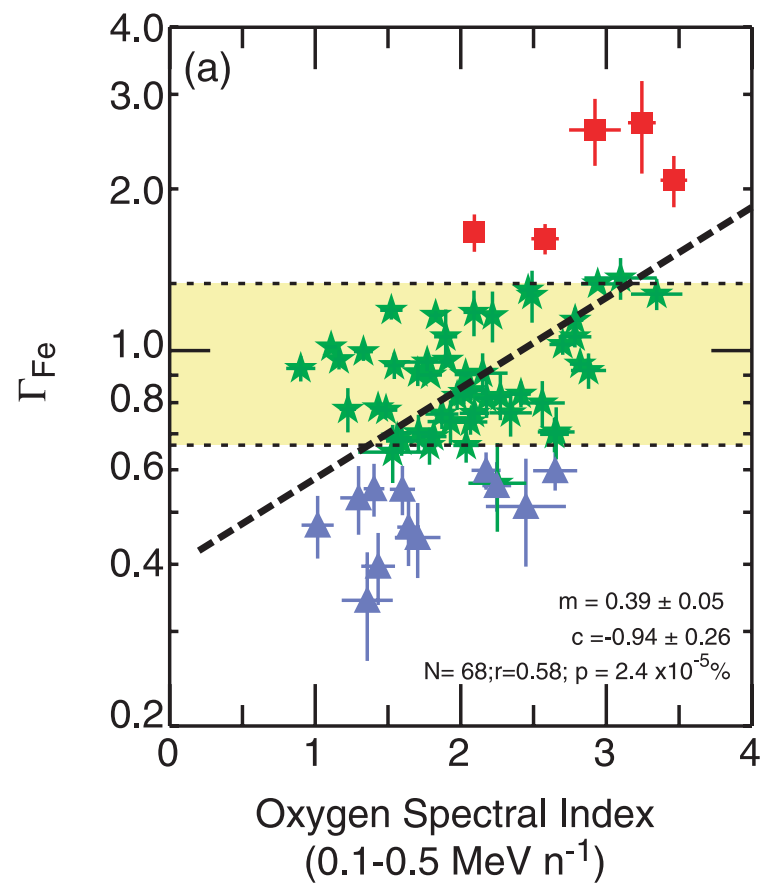

normalized to the ratios measured during the corresponding ambient intervals. The normalized $\mathrm{C} / \mathrm{O}$ ratios in all three events were close to unity, which implies that the $\mathrm{C} / \mathrm{O}$ ratios at the three IP shocks and in the ambient intervals were similar and had remained constant with energy. In contrast, the $\mathrm{Fe} / \mathrm{O}$ ratios at the shocks in all three events were depleted relative to the ambient values (also see Fig. 1). This is a general result for the entire survey: the $\mathrm{Fe} / \mathrm{O}$ ratio was depleted by an average of $\sim 30 \%$ at the shock compared with the ambient abundances. Note that for event 37 , which had $\mathrm{Fe} / \mathrm{O}$ increasing with energy, the shock/ambient $\mathrm{Fe} / \mathrm{O}$ ratios are also constant in energy. This is because the increase in $\mathrm{Fe} / \mathrm{O}$ at this shock was also present in the ambient spectrum, and when the shock Fe/ $\mathrm{O}$ was divided by the ambient $\mathrm{Fe} / \mathrm{O}$, a ratio constant with energy results even though both input terms individually increased with energy.

\subsection{Relative Behavior of $\mathrm{Fe}$ and $\mathrm{O}$ Spectra}

Figure 12 shows scatter plots of $(a)$ the $0.1-0.5 \mathrm{MeV}$ nucleon $^{-1} \mathrm{O}$ shock spectral indices $\gamma_{s}$ versus the spectral indices $\gamma_{a}$ measured during the ambient intervals, and $(b)$ shock values of $\Gamma_{\mathrm{Fe}}$ versus $\Gamma_{\mathrm{Fe}}$ for the ambient suprathermals. Figure $12 a$ shows that $\gamma_{s}$ was positively correlated with $\gamma_{a}$. Figure $12 b$ shows that the relative behavior of $\mathrm{Fe}$ and $\mathrm{O}$

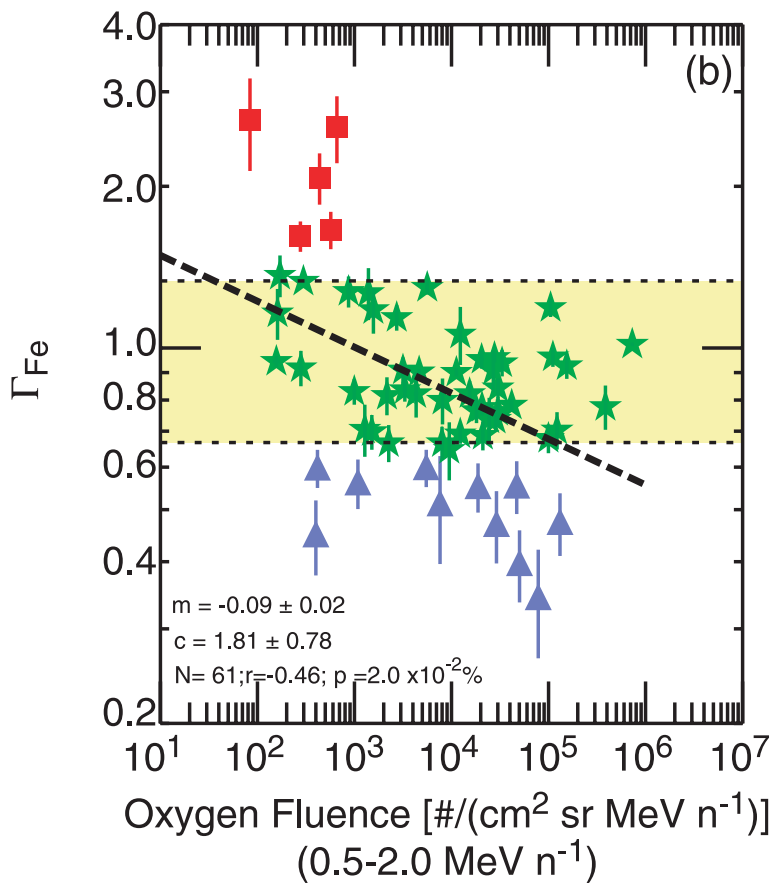

FIG. 8. $-\Gamma_{\mathrm{Fe}}$ vs. (a) the $0.1-0.5 \mathrm{MeV}$ nucleon ${ }^{-1} \mathrm{O}$ spectral indices and $(b)$ the $0.5-2.0 \mathrm{MeV}$ nucleon ${ }^{-1} \mathrm{O}$ fluences. The dashed lines show the linear fit to the data. The terms $m$ and $c$ are the slope and intercept of the fit. The yellow band identifies events where Fe/O remained constant with energy within $\pm 33 \%$. Seven intense events in which ULEIS saturated are not shown in $(b)$. 

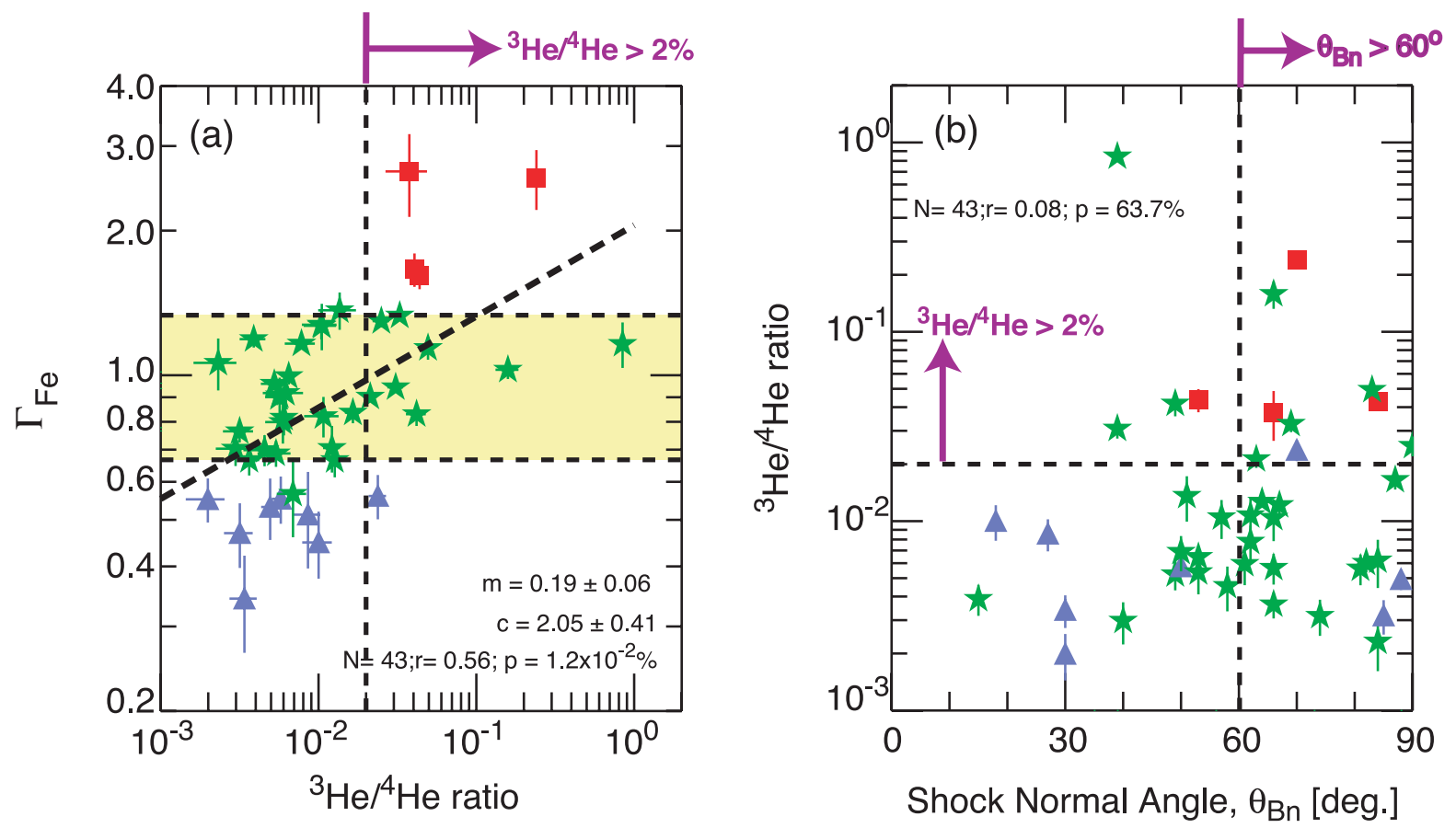

FIG. 9. - (a) $\Gamma_{\mathrm{Fe}}$ vs. the $0.5-2.0 \mathrm{MeV}$ nucleon ${ }^{-1}{ }^{3} \mathrm{He} /{ }^{4} \mathrm{He}$ ratio and $(b){ }^{3} \mathrm{He} /{ }^{4} \mathrm{He}$ ratio vs. $\theta_{B n}$ for $43{ }^{3} \mathrm{He}$-rich IP shocks (identified in Desai et al. 2003).

spectra was also reasonably well correlated with that measured during the ambient intervals. Note that for three IP shock events for which $\mathrm{Fe} / \mathrm{O}$ had increased with energy (red symbols), in each case $\mathrm{Fe} / \mathrm{O}$ had increased with energy in the ambient population.

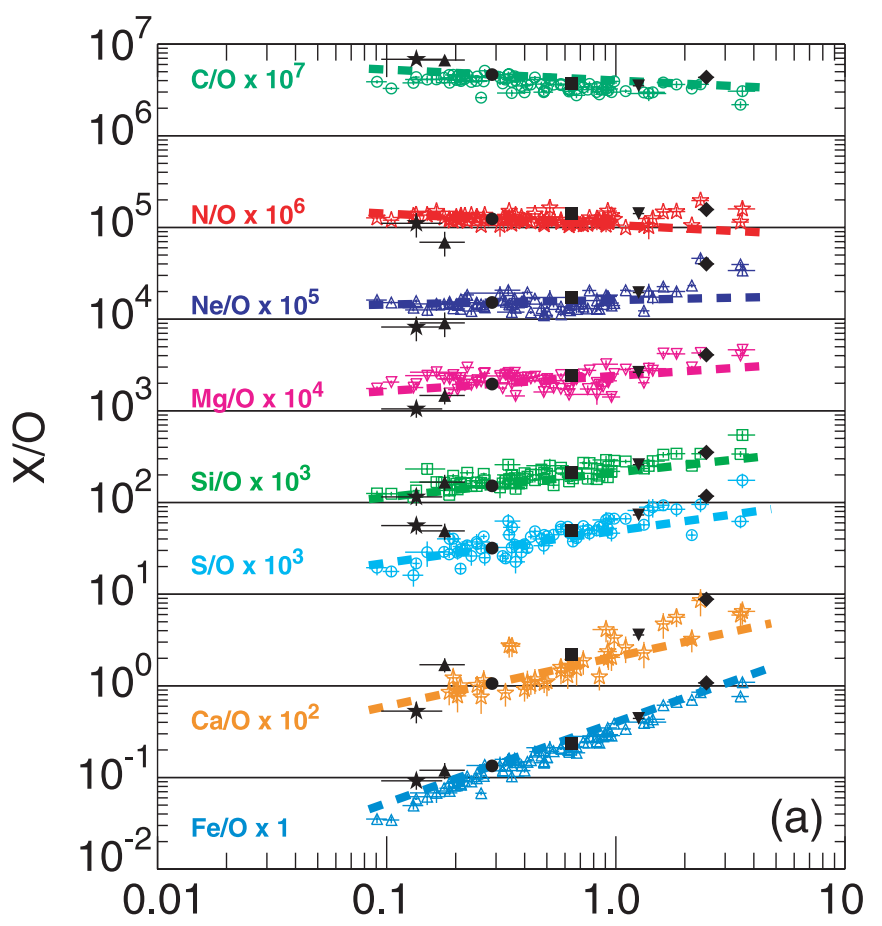

$\mathrm{Fe} / \mathrm{C}$

\section{SUMMARY OF OBSERVATIONS}

The main results of our survey of the spectral properties of 0.1-100 MeV nucleon ${ }^{-1} \mathrm{C}, \mathrm{O}$, and Fe nuclei associated with 72 IP shock events are as follows:

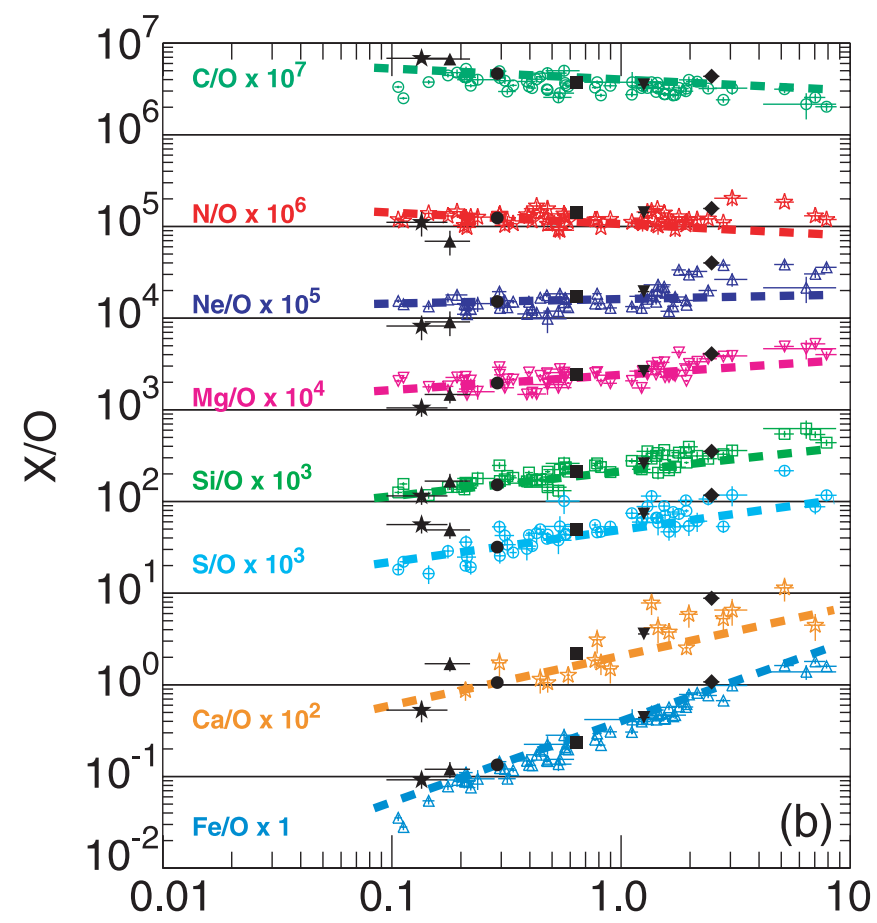

$\mathrm{Fe} / \mathrm{C}$

Fig. 10.- The $0.32-0.45 \mathrm{MeV}$ nucleon ${ }^{-1}$ elemental abundances X/O vs. Fe/C measured during $(a)$ the shock-associated and $(b)$ the ambient sampling intervals. The solid black symbols represent average abundances in reference populations taken from Table 3 of Desai et al. (2003) as follows: stars, slow solar wind; triangles, fast solar wind; circles, gradual SEP events; squares, IP shock event average; inverted triangles, ambient suprathermals; diamonds, impulsive SEP events. The dashed curves are given by eq. (1) and are normalized to the gradual SEP event values. 

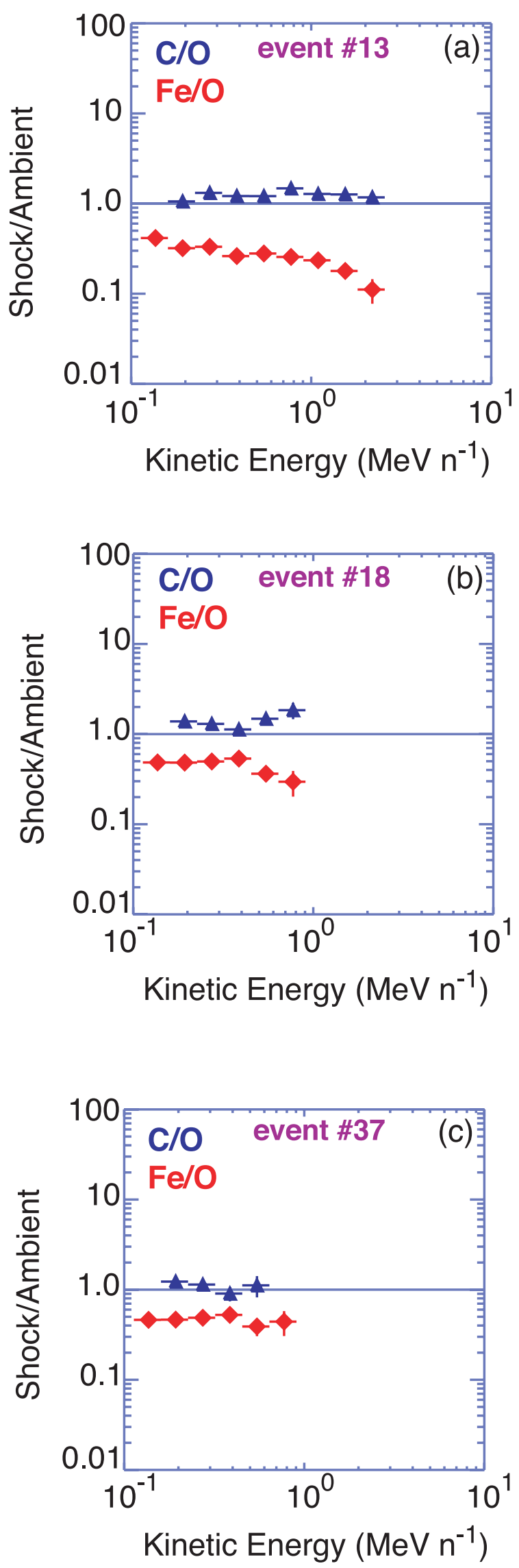

FIG. 11.- $\mathrm{C} / \mathrm{O}$ and $\mathrm{Fe} / \mathrm{O}$ ratios measured during IP shock events 13,18 , and 37 normalized to the corresponding ambient values, plotted vs. energy.
1. Figure 5 shows that the $0.1-0.5 \mathrm{MeV}$ nucleon $^{-1} \mathrm{O}$ spectral indices are very closely correlated with those of $\mathrm{C}$ $(r=0.98)$ and well correlated those of Fe $(r=0.79)$.

2. The $\mathrm{O}$ spectral index $\gamma$ and $e$-folding energy $E_{0}$ exhibit no clear dependence on the Mach number $M_{A}$, the magnetic $M$ and density $H$ compression ratios, the shock normal angle $\theta_{B n}$, and the shock speeds $V_{\mathrm{IP}}$ and $V_{S}$ in the spacecraft and upstream plasma frames (e.g., Figs. 6 and 13).

3. About $7 \%$ of the events exhibit rising $\mathrm{Fe} / \mathrm{O}$ ratios with energy, whereas the $\mathrm{Fe} / \mathrm{O}$ ratio in the remaining events either remains constant or decreases with increasing energy (Fig. 7).

4. Nine out of 12 events observed from 12 to $60 \mathrm{MeV}$ nucleon $^{-1}$ have $\mathrm{Fe} / \mathrm{O}$ decreasing with energy (Fig. 7c).

5. The energy dependence of $\mathrm{Fe} / \mathrm{O}$, defined as $\Gamma_{\mathrm{Fe}}$, is positively correlated with the $\sim 1 \mathrm{MeV}$ nucleon ${ }^{-1}{ }^{3} \mathrm{He} /{ }^{4} \mathrm{He}$ ratio; $\Gamma_{\mathrm{Fe}}$ is independent of shock strength parameters and $\theta_{B n}$ (Fig. 9).

6. The IP shock and ambient suprathermal ion abundances exhibit similar fractionation patterns characterized by power laws in $(M / Q)^{\delta}$ when compared with average gradual SEP abundances (Fig. 10).

7. The $\mathrm{Fe} / \mathrm{O}$ ratio during the shock events is typically $\sim 30 \%$ lower than the ambient $\mathrm{Fe} / \mathrm{O}$ ratio (Figs. 1 and 11).

8. Both the $\mathrm{O}$ spectral indices and the energy dependence of $\mathrm{Fe} / \mathrm{O}$ at the IP shocks are well correlated with those of the ambient suprathermals (Fig. 12).

\section{DISCUSSION}

\subsection{Implications for Shock Acceleration Models}

Van Nes et al. (1984) had reported that the spectral indices $\gamma$ of $\sim 0.035-0.24 \mathrm{MeV}$ protons for $\sim 75 \%$ of the IP shocks observed at ISEE 3 fell within the $\pm 25 \%$ limits of the predicted relationship between $\gamma$ and the density compression ratio $H$, where in a simple, one-dimensional steady state theory the spectral index $\gamma=(H+2) /(2 H-2)$. For the shocks studied here, Figure 13 plots the $\mathrm{O}$ spectral indices $\gamma$ averaged over $2 \mathrm{hr}$ intervals centered on shock passages versus $(M+2) /(2 M-2)$, where $M$ is the magnetic compression ratio. The figure clearly shows that the shock spectra were poorly correlated with the compression ratio, although the uncertainties are large enough that the $\gamma$-values of 35 events (i.e., $\sim 58 \%$ ) fell within the $\pm 25 \%$ limits (also see Ho et al. 2003). Finally, we note that 18 of the remaining 25 events that fell outside the error limits of $M$ had softer spectra than the theoretical prediction (also see Kallenrode 1995; Ho et al. 2003), which essentially implies that steady state theory overestimates the acceleration efficiency of many IP shocks in our survey.

Our analysis of the energy spectra of the shock-associated heavy ions has yielded several important results. First, even though the energy spectra measured by ULEIS were well represented by a power law multiplied by an exponential, the fits to the combined ULEIS and SIS spectra were visually unsatisfactory and had very high $\chi^{2}$ values, which indicates that the Jones \& Ellison expression of $j(E)=$ $j_{0} E^{-\gamma} \exp \left(-E / E_{0}\right)$ was unable to represent the shockassociated spectrum over a broad energy range (see also Tylka et al. 2000; Mewaldt et al. 2003). Second, the relatively poor correspondence between the $\mathrm{Fe}$ and $\mathrm{O}$ spectral indices is somewhat at odds with shock acceleration models that inject monoenergetic seed populations and produce similar $\gamma$-values for all species. Finally, from the results shown in Figures 5, 6, 

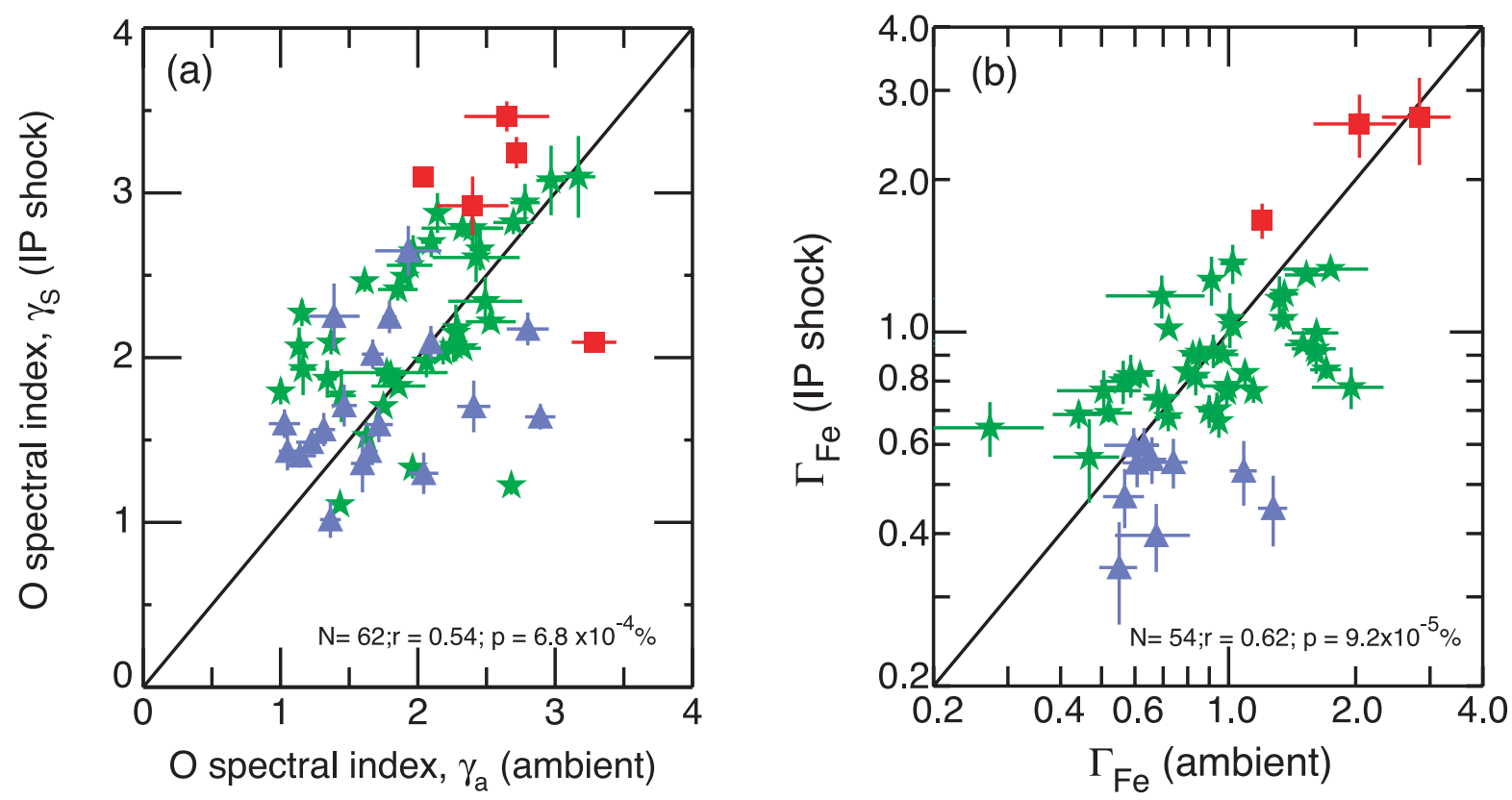

FIG. 12. (a) $\mathrm{O}$ spectral index at IP shocks vs. that for the ambient suprathermals. (b) $\Gamma_{\mathrm{Fe}}$ for IP shocks vs. $\Gamma_{\mathrm{Fe}}$ measured during the ambient intervals.

9 , and 13 , we conclude that the locally measured characteristics of the IP shocks played no significant role either in determining the spectral properties or in producing the event-to-event variability during our survey, and that steady state theory cannot be used to predict the behavior of the IP shock-associated heavy ions at $A C E$.

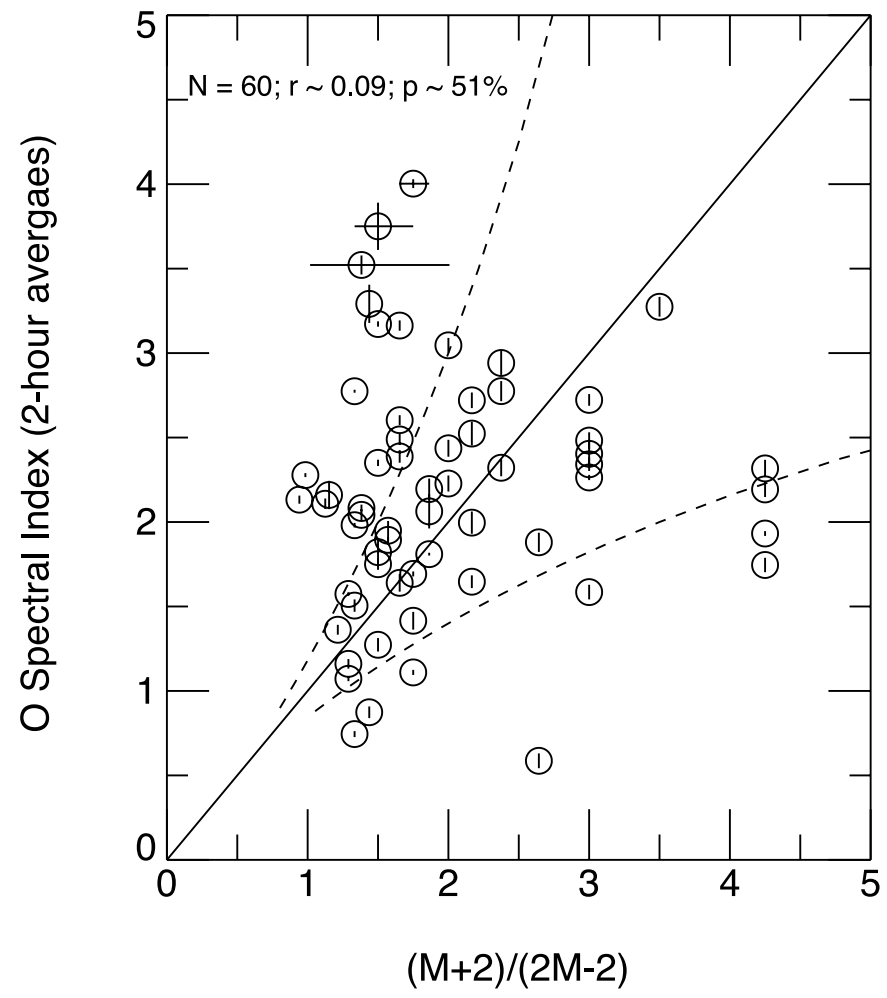

FIG. 13.-Scatter plot of the $0.1-0.5 \mathrm{MeV}$ nucleon $^{-1} \mathrm{O}$ spectral indices $\gamma$ obtained over $2 \mathrm{hr}$ intervals centered on shock passage vs. $(M+2) /(2 M-2)$; $M$ is the magnetic compression ratio. Typical error bars for $(M+2) /(2 M-2)$ are shown for a few points. Solid line: Relationship between $\gamma$ and $M$ as predicted by steady state theory. Dashed curves: The $\pm 25 \%$ error limits of $M$. Events that lie above the top curve have softer spectra than predicted.
Of course it is possible that the energetic particles measured during our sampling intervals were accelerated at different locations on the shock front or at earlier times when the shock properties may have been significantly different from those determined at $A C E$, thereby contributing to the lack of clear relationships between the local shock parameters and the spectral fit parameters. Indeed, significantly different values for $\theta_{B n}$ have been observed for the same IP shock at Wind and $A C E$ that were separated by less than $\sim 100 R_{E}$ (Szabo $2001)$. It is therefore conceivable that the contemporary twodimensional time-dependent numerical models of particle acceleration and transport at evolving IP shocks (e.g., Lario et al. 1998; Zank et al. 2000; Li et al. 2003) might be able to account for some of the above spatial and temporal effects. Nonetheless, we believe that in addition to the results of our earlier two surveys (see Desai et al. 2001, 2003), the following results presented here will also pose serious challenges for both steady state and time-dependent models because most such models inject and accelerate a seed population of solar wind ions. These include (1) the relatively weak correspondence between the $\mathrm{Fe}$ and $\mathrm{O}$ spectral indices, (2) the poor correlation between the IP shock and solar wind abundances, and (3) the increase in $\mathrm{Fe} / \mathrm{O}$ with energy in $\sim 7 \%$ of the events.

\subsection{Can Shock Acceleration Processes Increase $\mathrm{Fe} / \mathrm{O}$ with Energy?}

The events where Fe/O increased with energy are difficult to understand in terms of rigidity-dependent acceleration mechanisms where ions with higher $M / Q$ are accelerated less efficiently than those with lower $M / Q$ (e.g., Lee 1983; Jones \& Ellison 1991; Zank et al. 2000; Li et al. 2003; Ruffolo \& Channok 2003). Recently, however, Cohen et al. (2003a, 2003b) used simple diffusion theory (after Ellison \& Ramaty 1985 ) to successfully model the energy-dependent behavior of $\mathrm{Fe} / \mathrm{O}$ above $\sim 12 \mathrm{MeV}$ nucleon ${ }^{-1}$ during 36 large gradual SEP events. Specifically, Cohen et al. (2003a, 2003b) showed that ratios such as $\mathrm{Fe} / \mathrm{O}$ might increase with energy, be constant, or decrease depending on the chosen form of magnetic field wave power spectrum. 
Even though the Cohen et al. (2003b) SEP survey differs from ours because they specifically excluded time intervals around the passage of IP shocks at $A C E$, there still might be common mechanisms at work. Thus it is possible that the Cohen et al. (2003a, 2003b) mechanism could account for the puzzling increase in $\mathrm{Fe} / \mathrm{O}$ with energy observed during the five IP shocks in our survey. However, we remark that neither this nor other mechanisms where higher rigidity ions are accelerated more efficiently than lower rigidity ions (e.g., Ellison 1985) could simultaneously account for some of the other observational features of most of these events, namely, the enrichments of high charge state $\mathrm{Fe}$ at $\sim 0.5 \mathrm{MeV}$ nucleon $^{-1}$ (Popecki et al. 2001) and that of the $\sim 1 \mathrm{MeV}$ nucleon $^{-1}$ ${ }^{3} \mathrm{He} /{ }^{4} \mathrm{He}$ ratio.

Other approaches to model odd compositional features have recently emphasized the role of the seed population. For example, in order to account for the ${ }^{3} \mathrm{He}$-enrichments above $\sim 15 \mathrm{MeV}^{\text {nucleon }}{ }^{-1}$ in four large gradual SEP events, Kocharov \& Torsti (2003) have recently modeled the reacceleration of $\sim 1 \mathrm{MeV}$ nucleon ${ }^{-1}{ }^{3} \mathrm{He}$ ions originating in impulsive flares by CME-driven shocks (after Mason et al. 1999a). Tylka et al. (2001) also used properties of the seed population in order to model the unusual behavior of the $\mathrm{Fe}$ spectrum above $\sim 20 \mathrm{MeV}$ nucleon $^{-1}$ in a large gradual SEP event: they assumed that the CME-driven shock accelerated a seed population of impulsive flare material enriched in high charge state Fe ions. Clearly then, one could fit our observations of IP shock abundances and spectra using a combination of processes where the ${ }^{3} \mathrm{He}$ and high charge state $\mathrm{Fe}$ enhancements occurred due to the reacceleration of impulsive flare material, while the increase in $\mathrm{Fe} / \mathrm{O}$ with energy occurred because of the types of processes described by Ellison (1985) or Cohen et al. (2003a, 2003b).

Thus, although strictly speaking we cannot rule out the possibility that the increase in $\mathrm{Fe} / \mathrm{O}$ with energy might have occurred because of some poorly understood aspect of the diffusive shock acceleration process, we remark that a thorough investigation of the occurrence of complex scenarios such as the one outlined above requires detailed case studies and modeling, which is well beyond the scope of this survey. Alternatively, however, on the basis of the evidence presented here and in Desai et al. $(2001,2003)$, we suggest below that the increase in $\mathrm{Fe} / \mathrm{O}$ ratio with energy in these events occurs primarily because of the acceleration of suprathermal seed spectra, which themselves have rising $\mathrm{Fe} / \mathrm{O}$ ratios with energy. This relatively simpler scenario essentially eliminates the necessity to invoke $M / Q$-dependent acceleration mechanisms where higher rigidity ions are accelerated more efficiently than the lower rigidity ions.

\subsection{Rigidity-dependent Acceleration of Seed Spectra by Interplanetary Shocks}

Previously we showed that the $\sim 1 \mathrm{MeV}$ nucleon $^{-1} \mathrm{Fe} / \mathrm{O}$ ratio at the 72 IP shocks was well correlated with the corresponding ratio measured during the ambient sampling intervals (Desai et al. 2003). On the basis of significantly large enrichments (up to a factor of $\sim 2000$ ) in the $\sim 1 \mathrm{MeV}$ nucleon $^{-1}$ ${ }^{3} \mathrm{He} /{ }^{4} \mathrm{He}$ ratio over the slow solar wind value of $\sim(4.08 \pm$ $0.25) \times 10^{-4}$ (Gloeckler \& Geiss 1998) and the relative abundances of ${ }^{4} \mathrm{He}-\mathrm{Fe}$ nuclei measured during the 72 events, we concluded that the seed population for these IP shocks was composed predominantly of suprathermal ions that were previously accelerated in impulsive and gradual SEP events in-

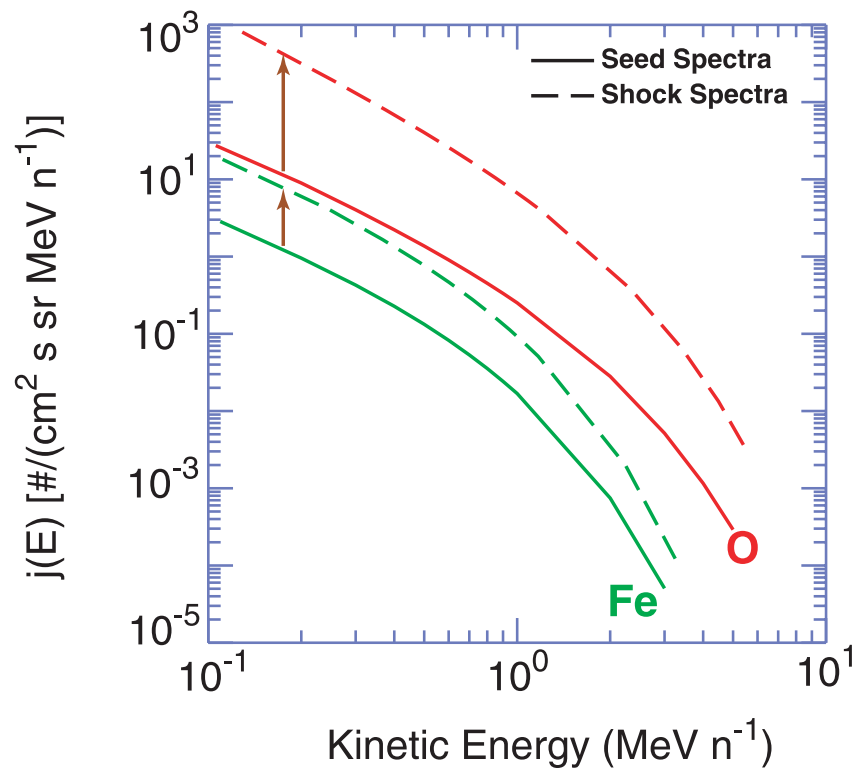

FIG. 14.- Sketch of average properties of the acceleration of $\mathrm{O}$ and Fe seed spectra, as inferred from the survey (see text).

stead of thermal or suprathermal solar wind ions (Desai et al. 2001, 2003). We also found that, on average, the IP shocks accelerated lower rigidity ions more efficiently than higher rigidity ions.

In this work, we have shown that the $\mathrm{O}$ spectral indices and the relative behavior of $\mathrm{Fe}$ and $\mathrm{O}$ spectra during the IP shock events were remarkably similar to those of the ambient suprathermal ions. The fact that $\mathrm{C} / \mathrm{O}$ remained constant with energy in these events while $\mathrm{Fe} / \mathrm{O}$ decreased with energy in the majority of the events further indicates that $\mathrm{C}$ and $\mathrm{O}$ were accelerated with more or less equal efficiency while $\mathrm{Fe}$ was accelerated less efficiently than both $\mathrm{C}$ and $\mathrm{O}$. In addition, the shock-associated $\mathrm{Fe} / \mathrm{O}$ ratio was depleted by $\sim 30 \%$ relative to the ambient particles, indicating that the less efficient acceleration of higher rigidity ions was observed on a case-by-case basis in addition to the average of the entire event sample.

However, an important question is whether the five IP shock events that exhibit an increase in $\mathrm{Fe} / \mathrm{O}$ with energy fit into the above picture? It was noted previously that a similar increase in $\mathrm{Fe} / \mathrm{O}$ with energy was also observed in the associated ambient populations in three of the five cases (two events did not have a measured ambient population). We therefore suggest that these seemingly unusual events can be understood if they also simply accelerated seed spectra composed of interplanetary suprathermal ions with $M / Q$-dependent processes similar to those of the other events in the survey. The fact that the $\sim 1 \mathrm{MeV}$ nucleon ${ }^{-1}{ }^{3} \mathrm{He} /{ }^{4} \mathrm{He}$ ratio during four of these five events was greater than $2 \%$ while that in the ambient intervals corresponding to three events was greater than $1 \%$ (not shown) further indicates that the source population for these events was probably dominated by material accelerated in impulsive SEP events (see Mason et al. 1999a; Desai et al. 2001; Tylka et al. 2001). The additional property of these five events, that they were all weak particle events (see Fig. 8), does not play any obvious role in this scenario.

A sketch of the results of reacceleration of an energetic seed particle distribution at IP shocks is shown in Figure 14, where the $\mathrm{Fe}$ and $\mathrm{O}$ source spectra are both shifted up in intensity 
after acceleration but the $M / Q$-dependent mechanisms identified by Desai et al. (2003) cause a larger increase in the $\mathrm{O}$ intensity than in the $\mathrm{Fe}$ one.

It is important to note that when compared with the average gradual SEP abundances, the abundances in individual gradual and impulsive SEP events are enhanced or depleted according to a power law in $(M / Q)^{\delta}$ (e.g., Breneman \& Stone 1985; Cohen et al. 1999; Leske et al. 1999; Slocum et al. 2003; Mason et al. 2004). This fractionation is believed to occur because of injection, acceleration, and/or transport processes that depend on ion rigidity (e.g., Cohen et al. 1999; $\mathrm{Ng}$ et al. 2003; Mason et al. 2004). Our results have clearly shown that the fractionation of elemental abundances during the shockassociated and ambient intervals was similar to that measured in gradual SEP events, but quite different when compared with the solar wind abundances. This is not surprising given that the ambient suprathermal ion population was composed of material accelerated previously in impulsive and gradual SEP events and that the IP shocks studied here simply reaccelerated these interplanetary suprathermals (see also Klecker et al. 1981; Hovestadt et al. 1982; Tan et al. 1989; Mason 2000; Desai et al. 2003).

Finally, since most current models have focused on injecting and accelerating solar wind ions at IP shocks (e.g., Lee 2000; Li et al. 2003), the reacceleration of energetic particle seed spectra has to date been explored only in a very limited fashion (Scholer \& Morfill 1975; Blandford \& Ostriker 1978; Axford 1981; Forman \& Webb 1985; Lee \& Ryan 1986; Jones \& Ellison 1991; Ruffolo \& Channok 2003). However, the results presented here and in Desai et al. $(2001,2003)$ have clearly shown that models injecting a stable monoenergetic seed population of solar wind ions are not able to account for the heavy-ion compositional and spectral variability of the CME-driven IP shock-associated ion populations. Thus, to understand the physics of shock acceleration using the new measurements at $A C E$, we suggest that models take account of the dynamic and variable nature of the seed population and inject realistic seed particle distributions composed of suprathermal ions originating in impulsive and gradual SEP events.

We are grateful to the members of the Space Physics Group, University of Maryland, and the Johns Hopkins Applied Physics Laboratory for the construction of the ULEIS instrument. We thank Adam Szabo for providing the shock analysis program. Work at the University of Maryland was supported by NASA contract NAS5-30927 and NASA grant PC 251428. Funding for C. W. S. and Q. H. is provided by CIT subcontract PC251439 under NASA grant NAG5-6912 for support of the $A C E$ magnetic field experiment. Work at Los Alamos was performed under the auspices of the US Department of Energy with financial support from the NASA $A C E$ project. We acknowledge the use of the lists of Solar Proton Events Affecting the Earth Environment provided by NOAA Space Environment Services Center at http://sec.noaa.gov/ftpdir/ indices/2001_events/20011122events.txt and that of CMEs provided by the $S O H O$ LASCO Team at ftp://lasco6.nascom. nasa.gov/pub/lasco/status/LASCO_CME_List_2001.

\section{APPENDIX}

Table A1 lists the shock parameters for the 72 IP shocks in this survey.

TABLE Al

Shock Parameters for the 72 Interplanetary Shocks in This Survey

\begin{tabular}{cccccccc}
\hline \hline & Shock Arrival Time & & $\theta_{B n}$ & & $V_{\text {IP }}$ & $V_{S}$ \\
Number & at $A C E$ (UT) & $M_{A}$ & $(\mathrm{deg})$ & $H$ & $M$ & $\left(\mathrm{~km} \mathrm{~s}^{-1}\right)$ & $\left(\mathrm{km} \mathrm{s}^{-1}\right)$ \\
$(1)$ & $(2)$ & $(3)$ & $(4)$ & $(5)$ & $(6)$ & $(7)$ & $(8)$ \\
\hline
\end{tabular}

1997

\begin{tabular}{|c|c|c|c|c|c|c|c|}
\hline $1 \ldots \ldots \ldots \ldots \ldots \ldots \ldots$ & Nov 22, 0906 & $\ldots$ & $\ldots$ & $\ldots$ & $\ldots$ & $\ldots$ & $\ldots$ \\
\hline \multicolumn{8}{|c|}{1998} \\
\hline $2^{\mathrm{a}}$ & Jan 28,1544 & $\ldots$ & $53 \pm 5$ & & $1.4 \pm 0.04$ & $\ldots$ & \\
\hline $3^{\mathrm{a}} \ldots \ldots \ldots \ldots \ldots \ldots$ & May 29,1504 & $\ldots$ & $63 \pm 3$ & $1.7 \pm 0.2$ & $1.5 \pm 0.02$ & $615 \pm 12$ & $73 \pm 9$ \\
\hline $4^{\mathrm{a}} \ldots \ldots \ldots \ldots \ldots \ldots$ & Jun 17, 2046 & $\ldots$ & $74 \pm 2$ & $\ldots$ & $1.3 \pm 0.01$ & $329 \pm 2$ & $\ldots$ \\
\hline $5 \ldots \ldots \ldots \ldots \ldots$ & Aug 6, 0644 & 1.5 & $82 \pm 3$ & $1.8 \pm 0.1$ & $1.8 \pm 0.1$ & $454 \pm 53$ & $84 \pm 12$ \\
\hline $6 \ldots \ldots \ldots \ldots \ldots \ldots$ & Sep 24,2313 & 3.2 & $62 \pm 2$ & $2.7 \pm 0.1$ & $2.5 \pm 0.1$ & $604 \pm 47$ & $341 \pm 20$ \\
\hline 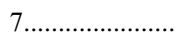 & Oct 18,1901 & 3.7 & $130 \pm 19$ & $2.2 \pm 0.3$ & $1.9 \pm 1.0$ & $332 \pm 45$ & $84 \pm 18$ \\
\hline 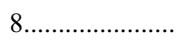 & Nov 30,0418 & 2.2 & $56 \pm 11$ & $2.8 \pm 0.4$ & $2.7 \pm 1.2$ & $416 \pm 37$ & $92 \pm 23$ \\
\hline 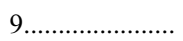 & Dec 1, 0253 & 1.7 & $103 \pm 14$ & $1.7 \pm 0.5$ & $1.7 \pm 0.8$ & $391 \pm 62$ & $68 \pm 6$ \\
\hline $10 \ldots \ldots$ & Dec 26, 0934 & 1.1 & $82 \pm 6$ & $1.4 \pm 0.1$ & $1.4 \pm 0.3$ & $555 \pm 74$ & $72 \pm 8$ \\
\hline
\end{tabular}

1999

\begin{tabular}{|c|c|c|c|c|c|c|c|}
\hline $11 \ldots \ldots \ldots \ldots \ldots \ldots$ & Feb 18, 0209 & 3.4 & $130 \pm 3$ & $2.9 \pm 0.1$ & $2.5 \pm 0.2$ & $671 \pm 26$ & $278 \pm 22$ \\
\hline $12 \ldots \ldots \ldots \ldots \ldots \ldots$ & May 5, 1459 & 1.9 & $39 \pm 30$ & $3.4 \pm 0.4$ & $4.1 \pm 4.0$ & $170 \pm 40$ & $53 \pm 23$ \\
\hline $13 \ldots \ldots \ldots \ldots \ldots \ldots$ & Jun 26, 1925 & 2.0 & $50 \pm 9$ & $2.3 \pm 0.1$ & $2.2 \pm 0.8$ & $444 \pm 44$ & $130 \pm 18$ \\
\hline $14 \ldots \ldots \ldots \ldots \ldots \ldots \ldots$ & Jul 2, 0024 & 1.8 & $69 \pm 5$ & $2.0 \pm 0.1$ & $2.0 \pm 0.3$ & $558 \pm 82$ & $139 \pm 8$ \\
\hline $15 \ldots \ldots \ldots \ldots \ldots \ldots$ & Jul 6, 1417 & 3.6 & $141 \pm 6$ & $2.4 \pm 0.9$ & $1.8 \pm 0.5$ & $554 \pm 119$ & $260 \pm 3$ \\
\hline $16 \ldots$ & Sep 12, 0321 & 4.1 & $113 \pm 4$ & $2.5 \pm 0.2$ & $2.3 \pm 0.2$ & $520 \pm 48$ & $161 \pm 19$ \\
\hline $17 \ldots \ldots \ldots$ & Sep 15, 1942 & 2.0 & $66 \pm 8$ & $2.1 \pm 0.3$ & $2.1 \pm 1.0$ & $590 \pm 48$ & $90 \pm 18$ \\
\hline $18 \ldots \ldots \ldots \ldots \ldots \ldots \ldots$ & Sep 22, 1146 & 2.7 & $64 \pm 7$ & $2.4 \pm 0.5$ & $2.3 \pm 0.3$ & $468 \pm 40$ & $131 \pm 17$ \\
\hline
\end{tabular}


TABLE A1-Continued

\begin{tabular}{|c|c|c|c|c|c|c|c|}
\hline $\begin{array}{l}\text { Number } \\
\text { (1) }\end{array}$ & $\begin{array}{c}\text { Shock Arrival Time } \\
\text { at } A C E(\mathrm{UT}) \\
\text { (2) }\end{array}$ & $\begin{array}{l}M_{A} \\
\text { (3) }\end{array}$ & $\begin{array}{c}\theta_{B n} \\
(\mathrm{deg}) \\
(4)\end{array}$ & $\begin{array}{l}H \\
(5)\end{array}$ & $\begin{array}{l}M \\
(6)\end{array}$ & $\begin{array}{c}V_{\mathrm{IP}} \\
\left(\mathrm{km} \mathrm{s}^{-1}\right) \\
(7)\end{array}$ & $\begin{array}{c}V_{S} \\
\left(\mathrm{~km} \mathrm{~s}^{-1}\right) \\
(8)\end{array}$ \\
\hline 19 ............................ & Oct 21,0138 & 1.4 & $77 \pm 3$ & $2.5 \pm 0.1$ & $2.5 \pm 0.3$ & $408 \pm 40$ & $83 \pm 19$ \\
\hline $20 \ldots \ldots \ldots \ldots \ldots \ldots$ & Dec 11,1201 & 1.3 & $116 \pm 40$ & $2.0 \pm 0.7$ & $2.1 \pm 4.2$ & $435 \pm 113$ & $49 \pm 21$ \\
\hline $21 \ldots \ldots \ldots \ldots \ldots \ldots \ldots$ & Dec 12, 1514 & 1.7 & $70 \pm 10$ & $2.4 \pm 0.3$ & $2.5 \pm 0.7$ & $475 \pm 134$ & $178 \pm 29$ \\
\hline \multicolumn{8}{|c|}{2000} \\
\hline$\ldots \ldots \ldots$ & Jan 22,0022 & 2.3 & $49 \pm 25$ & $1.8 \pm 0.1$ & $1.6 \pm 0.9$ & $182 \pm 64$ & $96 \pm 11$ \\
\hline $23 \ldots \ldots \ldots \ldots \ldots \ldots$ & Feb 11, 0213 & 1.8 & $153 \pm 16$ & $2.2 \pm 0.3$ & $1.9 \pm 0.6$ & $484 \pm 44$ & $103 \pm 19$ \\
\hline 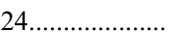 & Feb 11, 2319 & 3.6 & $92 \pm 4$ & $2.8 \pm 0.1$ & $2.8 \pm 0.2$ & $539 \pm 37$ & $224 \pm 27$ \\
\hline $25^{\mathrm{a}} \ldots \ldots \ldots \ldots \ldots \ldots$ & Feb 20, 2047 & $\ldots$ & $85 \pm 3$ & $2.2 \pm 0.2$ & $2.2 \pm 0.1$ & $413 \pm 6$ & $129 \pm 2$ \\
\hline $26 \ldots \ldots \ldots \ldots \ldots \ldots$ & Apr 24, 0852 & 1.3 & $70 \pm 9$ & $1.6 \pm 0.1$ & $1.6 \pm 0.3$ & $405 \pm 79$ & $132 \pm 6$ \\
\hline 27...................... & Jun 23,1227 & 3.5 & $66 \pm 2$ & $2.5 \pm 0.0$ & $2.4 \pm 0.1$ & $607 \pm 12$ & $217 \pm 9$ \\
\hline $28 \ldots \ldots \ldots \ldots \ldots \ldots$ & Jul 10, 0558 & 1.8 & $66 \pm 13$ & $2.1 \pm 0.2$ & $2.0 \pm 0.8$ & $483 \pm 50$ & $134 \pm 14$ \\
\hline $29 \ldots \ldots \ldots \ldots \ldots \ldots$ & Jul 11, 1123 & 1.1 & $96 \pm 3$ & $2.3 \pm 0.5$ & $2.3 \pm 0.5$ & $300 \pm 46$ & $58 \pm 18$ \\
\hline 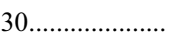 & Jul 13, 0919 & 1.4 & $158 \pm 13$ & $1.5 \pm 0.1$ & $1.4 \pm 0.5$ & $169 \pm 79$ & $113 \pm 4$ \\
\hline 31_..................... & Jul 19, 1449 & 3.0 & $99 \pm 7$ & $2.9 \pm 0.5$ & $2.8 \pm 0.5$ & $606 \pm 38$ & $150 \pm 27$ \\
\hline 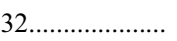 & Jul 26, 1755 & 1.6 & $84 \pm 4$ & $1.8 \pm 0.1$ & $1.8 \pm 0.2$ & $358 \pm 55$ & $44 \pm 10$ \\
\hline 33.......................... & Jul 28, 0910 & 1.6 & $129 \pm 8$ & $2.4 \pm 1.2$ & $2.6 \pm 1.9$ & $448 \pm 33$ & $119 \pm 24$ \\
\hline 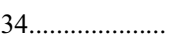 & Aug 10,0407 & 1.2 & $18 \pm 9$ & $2.4 \pm 0.3$ & $1.1 \pm 0.2$ & $429 \pm 39$ & $74 \pm 19$ \\
\hline 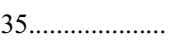 & Aug 11, 1811 & 1.7 & $61 \pm 5$ & $2.1 \pm 0.1$ & $2.0 \pm 0.3$ & $475 \pm 80$ & $245 \pm 22$ \\
\hline 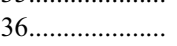 & Sep 6, 1613 & 2.3 & $85 \pm 3$ & $2.3 \pm 0.1$ & $2.3 \pm 0.1$ & $485 \pm 42$ & $131 \pm 18$ \\
\hline 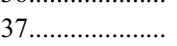 & Oct 5,0240 & 2.9 & $114 \pm 8$ & $2.4 \pm 0.1$ & $2.3 \pm 0.1$ & $517 \pm 49$ & $188 \pm 19$ \\
\hline 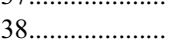 & Oct 12,2145 & 1.6 & $83 \pm 6$ & $2.5 \pm 1.3$ & $2.5 \pm 1.1$ & $448 \pm 34$ & $132 \pm 30$ \\
\hline 39....................... & Nov 4,0135 & 2.0 & $96 \pm 3$ & $2.9 \pm 0.1$ & $2.9 \pm 0.2$ & $373 \pm 34$ & $70 \pm 17$ \\
\hline 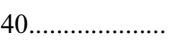 & Nov 26,0500 & 2.6 & $117 \pm 8$ & $1.6 \pm 0.1$ & $1.6 \pm 0.3$ & $433 \pm 79$ & $143 \pm 8$ \\
\hline 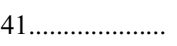 & Nov 28,0458 & 2.1 & $123 \pm 7$ & $2.3 \pm 0.2$ & $2.2 \pm 0.4$ & $534 \pm 49$ & $106 \pm 17$ \\
\hline \multicolumn{8}{|c|}{2001} \\
\hline $42 \ldots \ldots \ldots \ldots . . . . . . . .$. & Jan 23, 1007 & 2.8 & $177 \pm 41$ & $3.1 \pm 1.6$ & $1.0 \pm 0.8$ & $532 \pm 32$ & $165 \pm 38$ \\
\hline 43 & Mar 27, 1716 & 1.3 & $49 \pm 22$ & $1.9 \pm 0.5$ & $2.1 \pm 1.1$ & $471 \pm 48$ & $134 \pm 16$ \\
\hline $44^{\mathrm{a}}$ & Mar 31, 0023 & 1.0 & $53 \pm 4$ & $\ldots$ & $3.1 \pm 0.1$ & $550 \pm 2$ & $117 \pm 1$ \\
\hline 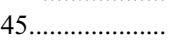 & Mar 31, 2256 & 1.1 & $87 \pm 5$ & $3.3 \pm 1.1$ & $3.3 \pm 1.1$ & $615 \pm 40$ & $145 \pm 27$ \\
\hline 46.................... & Apr 4,1422 & 4.2 & $15 \pm 5$ & $4.0 \pm 2.0$ & $1.6 \pm 0.5$ & $692 \pm 48$ & $305 \pm 24$ \\
\hline $47^{\mathrm{a}}$ & Apr 7, 1659 & $\ldots .2$ & $40 \pm 4$ & $1.9 \pm 0.3$ & $1.9 \pm 0.3$ & $542 \pm 3$ & $96 \pm 3$ \\
\hline 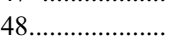 & Apr 8, 1033 & 4.2 & $90 \pm 3$ & $2.8 \pm 0.1$ & $2.8 \pm 0.2$ & $743 \pm 41$ & $254 \pm 25$ \\
\hline $49^{\mathrm{a}} \ldots \ldots \ldots \ldots \ldots$ & Apr 13, 0714 & $\ldots$ & $60 \pm 2$ & $1.3 \pm 0.1$ & $1.4 \pm 0.04$ & $748 \pm 10$ & $74 \pm 3$ \\
\hline $50 \ldots \ldots \ldots \ldots \ldots \ldots \ldots .$. & Apr 28, 0432 & 5.9 & $92 \pm 2$ & $3.7 \pm 0.8$ & $3.7 \pm 0.7$ & $905 \pm 59$ & $492 \pm 36$ \\
\hline $51^{\mathrm{a}} \ldots \ldots \ldots \ldots \ldots$ & Jun 18,0155 & $\ldots$ & $58 \pm 5$ & $\ldots$ & $1.6 \pm 0.04$ & $350 \pm 3$ & $\ldots$ \\
\hline 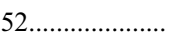 & Aug 17, 1016 & 2.7 & $118 \pm 6$ & $4.5 \pm 0.9$ & $4.4 \pm 1.2$ & $451 \pm 31$ & $138 \pm 31$ \\
\hline $53 \ldots \ldots \ldots \ldots \ldots \ldots$ & Aug 27, 1919 & 2.7 & $92 \pm 6$ & $2.8 \pm 0.7$ & $2.8 \pm 0.6$ & $486 \pm 39$ & $150 \pm 20$ \\
\hline 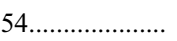 & Sep 14,0118 & 2.3 & $114 \pm 5$ & $2.6 \pm 0.5$ & $2.5 \pm 0.5$ & $490 \pm 46$ & $127 \pm 18$ \\
\hline 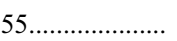 & Oct 11,1620 & 2.1 & $74 \pm 4$ & $2.7 \pm 0.1$ & $2.7 \pm 0.3$ & $540 \pm 35$ & $142 \pm 22$ \\
\hline $56 \ldots \ldots \ldots \ldots \ldots \ldots$ & Oct 21,1612 & 4.7 & $50 \pm 4$ & $2.5 \pm 0.5$ & $2.1 \pm 0.3$ & $587 \pm 39$ & $226 \pm 20$ \\
\hline 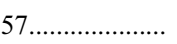 & Oct 25,0802 & 3.6 & $150 \pm 22$ & $3.8 \pm 0.6$ & $2.4 \pm 1.3$ & $406 \pm 34$ & $89 \pm 24$ \\
\hline 58 & Oct 28,0242 & 2.1 & $30 \pm 4$ & $2.7 \pm 0.2$ & $2.3 \pm 0.5$ & $470 \pm 45$ & $206 \pm 27$ \\
\hline $59^{\mathrm{a}} \ldots \ldots \ldots \ldots \ldots \ldots$ & Nov 6,0124 & $\ldots$ & $73 \pm 7$ & $\ldots$ & $2.8 \pm 0.04$ & $\ldots$ & $\ldots$ \\
\hline 60........................... & Nov 19,1735 & 3.0 & $65 \pm 4$ & $2.0 \pm 0.1$ & $1.9 \pm 0.2$ & $630 \pm 62$ & $196 \pm 8$ \\
\hline $61^{\mathrm{a}} \ldots \ldots \ldots \ldots \ldots$ & Nov 24,0538 & $\ldots$ & $56 \pm 9$ & $\ldots$ & $2.6 \pm 0.1$ & & $\ldots$ \\
\hline $62 \ldots \ldots \ldots \ldots \ldots \ldots$ & Dec 29,0448 & 2.9 & $138 \pm 9$ & $3.5 \pm 0.9$ & $2.9 \pm 0.6$ & $362 \pm 38$ & $120 \pm 26$ \\
\hline $63 \ldots \ldots \ldots \ldots \ldots$ & Dec 30, 1931 & 1.6 & $57 \pm 4$ & $2.4 \pm 0.1$ & $2.5 \pm 0.2$ & $459 \pm 42$ & $152 \pm 20$ \\
\hline \multicolumn{8}{|c|}{2002} \\
\hline 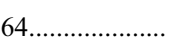 & Mar 18, 1237 & 6.1 & $38 \pm 6$ & $4.7 \pm 0.2$ & $3.1 \pm 0.6$ & $410 \pm 30$ & $98 \pm 22$ \\
\hline 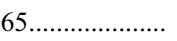 & Apr 17,1021 & 2.2 & $90 \pm 1$ & $3.4 \pm 0.1$ & $3.4 \pm 0.2$ & $460 \pm 30$ & $122 \pm 20$ \\
\hline 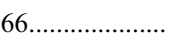 & Apr 19, 0803 & 1.6 & $67 \pm 6$ & $2.4 \pm 0.5$ & $2.5 \pm 0.6$ & $511 \pm 135$ & $275 \pm 22$ \\
\hline 67...................... & Apr 19, 2148 & 2.0 & $127 \pm 20$ & $1.2 \pm 0.1$ & $1.1 \pm 0.4$ & $566 \pm 163$ & $148 \pm 24$ \\
\hline 68........................... & May 18,1919 & 4.4 & $122 \pm 3$ & $3.0 \pm 0.1$ & $2.7 \pm 0.3$ & $470 \pm 42$ & $171 \pm 21$ \\
\hline 69.......................... & May 23,1016 & 4.2 & $96 \pm 2$ & $1.7 \pm 0.2$ & $1.7 \pm 0.2$ & $834 \pm 82$ & $466 \pm 4$ \\
\hline 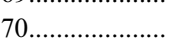 & Jul 17,1526 & 2.2 & $35 \pm 12$ & $2.6 \pm 0.2$ & $2.2 \pm 0.6$ & $493 \pm 40$ & $130 \pm 20$ \\
\hline 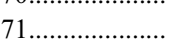 & Sep 7, 1609 & 2.4 & $89 \pm 4$ & $2.9 \pm 0.1$ & $2.9 \pm 0.2$ & $628 \pm 40$ & $231 \pm 28$ \\
\hline $72^{\mathrm{a}}$ & Sep 30,0721 & $\ldots$ & $39 \pm 2$ & $\ldots$ & $1.6 \pm 0.1$ & $\ldots$ & $\ldots$ \\
\hline
\end{tabular}

Notes.-Also see Desai et al. (2003). Col. (1): Number. Col. (2): Shock arrival time at $A C E$ (accurate to the nearest minute). Col. (3): Mach number. Col. (4): Shock normal angle. For 25 events with $\theta_{B n}>90^{\circ}$, this is given by $180-\theta_{B n}$. Col. (5): Density compression ratio $H=\rho_{D} / \rho_{U}$. Col. (6): Magnetic compression ratio $M=B_{D} / B_{U}$. Col. (7): Shock speed in spacecraft frame. Col. (8): Shock speed in upstream plasma frame.

${ }^{\mathrm{a}} \theta_{B n}$ determined using the magnetic coplanarity technique. 
Armstrong, T. P., Pesses, M. E., \& Decker, R. B. 1985, in Collisionless Shocks in the Heliosphere: Reviews of Current Research, ed. B. T. Tsurutani \& R. G. Stone (Geophys. Monogr. 35; Washington, DC: AGU), 271

Axford, I. 1981, in Proc. 17th Int. Cosmic Ray Conf. (Paris), 12, 155

Baring, M. G., et al. 1997, ApJ, 476, 889

Bevington, P. R., \& Robinson, D. K. 1992, Data Reduction and Error Analysis for the Physical Sciences (2nd ed.; New York: McGraw-Hill)

Blandford, R. D., \& Ostriker, J. P. 1978, ApJ, 221, L29

Breneman, H. H., \& Stone, E. C. 1985, ApJ, 299, L57

Cane, H. V., \& Richardson, I. G. 2003, J. Geophys. Res., 108, 1156, DOI: 10.1029/ 2002JA009817

Cohen, C. M. S., et al. 1999, Geophys. Res. Lett., 26, 149 . 2003a, Adv. Space Res., 32, 2649

2003b, in Proc. 28th Int. Cosmic Ray Conf. (Tsukuba), 6/7, 3241

Cummings, A. C., Stone, E. C., \& Steenberg, C. D. 2002, ApJ, 578, 194

Dalla, S., et al. 2003, Geophys. Res. Lett., 30(19), 8035, DOI: 10.1029/ 2003GL017139

Decker, R. B. 1988, Space Sci. Rev., 48, 195

Desai, M. I., et al. 1999, J. Geophys. Res., 104, 6705 . 2001, ApJ, 553, L89

2003, ApJ, 588, 1149

Eichler, D. 1981, ApJ, 244, 711

Ellison, D. C. 1985, J. Geophys. Res., 90, 29

Ellison, D. C., \& Ramaty, R. 1985, ApJ, 298, 400

Forman, M. A., \& Webb, G. M. 1985, in Collisionless Shocks in the Heliosphere: Reviews of Current Research, ed. B. T. Tsurutani \& R. G. Stone (Geophys. Monogr. 35; Washington, DC: AGU), 91

Gloeckler, G., \& Geiss, J. 1998, Space Sci. Rev., 84, 275

Gosling, J. T., et al. 1981, J. Geophys. Res., 86, 547

Ho, G. C., et al. 2003, in Proc. 28th Int. Cosmic Ray Conf. (Tsukuba), $6 / 7,3689$

Hovestadt, D., et al. 1982, ApJ, 258, L57

Jokipii, J. R. 1982, ApJ, 255, 716 1987, ApJ, 313, 842

Jones, F. C., \& Ellison, D. C. 1991, Space Sci. Rev., 58, 259

Kallenrode, M.-B. 1995, Adv. Space Res., 15(8/9), 375

Kennel, C. F., et al. 1986, J. Geophys. Res., 91, 11917

Klecker, B., et al. 1981, ApJ, 251, 393 1999, in Proc. 26th Int. Cosmic Ray Conf. (Salt Lake City), 6, 83 2000, in AIP Conf. Proc. 528, Acceleration and Transport of Energetic

Particles Observed in the Heliosphere, ed. R. A. Mewaldt, J. R. Jokipii,

M. A. Lee, E. Möbius, \& T. H. Zurbuchen (New York: AIP), 135 2003, in Proc. 28th Int. Cosmic Ray Conf. (Tsukuba), 6/7, 3277

Kocharov, L., \& Torsti, J. 2003, ApJ, 586, 1430

Lario, D., Sanahuja, B., \& Heras, A. M. 1998, ApJ, 509, 415

Lee, M. A. 1983, J. Geophys. Res., 88, 6109 2000, in AIP Conf. Proc. 528, Acceleration and Transport of Energetic

Particles Observed in the Heliosphere, ed. R. A. Mewaldt, J. R. Jokipii,

M. A. Lee, E. Möbius, \& T. H. Zurbuchen (New York: AIP), 227

Lee, M. A., \& Fisk, L. A. 1982, Space Sci. Rev., 32, 205

Lee, M. A., \& Ryan, J. M. 1986, ApJ, 303, 829

Leske, R. A., et al. 1999, Geophys. Res. Lett., 26, 153
Li, G., Zank, G. P., \& Rice, W. K. M. 2003, J. Geophys. Res., 108(A2), 1082, DOI: $10.1029 / 2002 J A 009666$

Mason, G. M. 2000, in AIP Conf. Proc. 528, Acceleration and Transport of Energetic Particles Observed in the Heliosphere, ed. R. A. Mewaldt, J. R. Jokipii, M. A. Lee, E. Möbius, \& T. H. Zurbuchen (New York: AIP), 234 Mason, G. M., Mazur, J. E., \& Dwyer, J. R. 1999a, ApJ, 525, L133

Mason, G. M., Mazur, J. E., Dwyer, J. R., Jokipii, J. R., Gold, R. E., \& Krimigis, S. M. 2004, ApJ, 606, 555

Mason, G. M., et al. 1996, Geophys. Res. Lett., 23, 1231 1998, Space Sci. Rev., 86, 409

1999b, Geophys. Res. Lett., 26, 141

McComas, D. J., et al. 1998, Space Sci. Rev., 86, 563

Mewaldt, R. A., et al. 2003, Eos, 84(3), SM52G-04

Möbius, E., et al. 1999, Geophys. Res. Lett., 26, 145 2000, in AIP Conf. Proc. 528, Acceleration and Transport of Energetic Particles Observed in the Heliosphere, ed. R. A. Mewaldt, J. R. Jokipii, M. A. Lee, E. Möbius, \& T. H. Zurbuchen (New York: AIP), 131

Ng, C. K., Reames, D. V., \& Tylka, A. J. 2003, ApJ, 591, 461

Popecki, M. A., et al. 2001, in Proc. 27th Int. Cosmic Ray Conf., 8, 3153

Reames, D. V. 1995, Adv. Space Res., 15(7), 41 1999, Space Sci. Rev., 90, 413

Richter, A. K., et al. 1985, in Collisionless Shocks in the Heliosphere: Reviews of Current Research, ed. B. T. Tsurutani \& R. G. Stone (Geophys. Monogr. 35; Washington, DC: AGU), 33

Ruffolo, D., \& Channok, C. 2003, in Proc. 28th Int. Cosmic Ray Conf. (Tsukuba), 6/7, 3681

Scholer, M. 1985, in Collisionless Shocks in the Heliosphere: Reviews of Current Research, ed. B. T. Tsurutani \& R. G. Stone (Geophys. Monogr. 35; Washington, DC: AGU), 287

Scholer, M., \& Morfill, G. 1975, Sol. Phys., 45, 227

Slocum, P. L., et al. 2003, ApJ, 594, 592

Smith, C. W., et al. 1998, Space Sci. Rev., 86, 613

Stone, E. C., et al. 1998a, Space Sci. Rev., 86, 1 1998b, Space Sci. Rev., 86, 357

Szabo, A. 1994, J. Geophys. Res., 99, 14737 2001, Eos, 82(47), F996

Tan, L. C., et al. 1989, ApJ, 345, 572

Tsurutani, B. T., \& Lin, R. P. 1985, J. Geophys. Res., 90, 1

Tylka, A. J., Reames, D. V., \& Ng, C. K. 1999, Geophys. Res. Lett., 26, 2141

Tylka, A. J., et al. 2000, in AIP Conf. Proc. 528, Acceleration and Transport of Energetic Particles Observed in the Heliosphere, ed. R. A. Mewaldt,

J. R. Jokipii, M. A. Lee, E. Möbius, \& T. H. Zurbuchen (New York: AIP), 147 2001, ApJ, 558, L59

van Nes, P., et al. 1984, J. Geophys. Res., 89, 2122

Viñas, A. F., \& Scudder, J. D. 1986, J. Geophys. Res., 91, 39

von Steiger, R., Geiss, J., \& Gloeckler, G. 1997, in Cosmic Winds and the

Heliosphere, ed. J. R. Jokipii, C. P. Sonnett, \& M. S. Giampapa (Tucson:

Univ. Arizona Press), 581

von Steiger, R., et al. 2000, J. Geophys. Res., 105, 27217

Wiedenbeck, M. E., et al. 2003, in AIP Conf. Proc. 679, Solar Wind Ten, ed.

M. Velli, R. Bruno, \& F. Malara (New York: AIP), 652

Zank, G. P., Rice, W. K. M., \& Wu, C. C. 2000, J. Geophys. Res., 105, 25079 